October 2000

DFUB 00-16

Version 3

hep-th/0010110

\title{
RELATIVE TOPOLOGICAL INTEGRALS AND RELATIVE CHEEGER-SIMONS DIFFERENTIAL CHARACTERS
}

\author{
by \\ Roberto Zucchini \\ Dipartimento di Fisica, Università degli Studi di Bologna \\ V. Irnerio 46, I-40126 Bologna, Italy \\ and \\ INFN, sezione di Bologna
}

\begin{abstract}
Topological integrals appear frequently in Lagrangian field theories. On manifolds without boundary, they can be treated in the framework of (absolute) (co)homology using the formalism of Cheeger-Simons differential characters. String and D-brane theory involve field theoretic models on worldvolumes with boundary. On manifolds with boundary, the proper treatment of topological integrals requires a generalization of the usual differential topological set up and leads naturally to relative (co)homology and relative CheegerSimons differential characters. In this paper, we present a construction of relative CheegerSimons differential characters which is computable in principle and which contains the ordinary Cheeger-Simons differential characters as a particular case.
\end{abstract}

PACS no.: 0240, 0460, 1110. Keywords: String Theory, Cohomology. 


\section{Introduction}

Topological integrals appear frequently in Lagrangian field theories such as ChernSimons model, Wess-Zumino-Witten model, gauge theory and D-brane theory, to mention only the most popular and best known. They are formal integrals on topologically non trivial manifolds of differential forms which are only locally defined. The integrand thus suffers ambiguities on overlapping coordinate patches, making the definition of integration problematic. In physics, the problem of the proper definition of topological integrals has been studied by several authors since the mid eighties $[1,2,3]$ and also recently it has been the object of a number of studies $[4,5,6,7,8]$. In mathematics, the interest in this topic dates back at least to the early seventies when it was attempted to frame the Chern-Simons forms associated to connections on a principal bundle in appropriate global differential topological structures on its base space. It resulted in the theory of Cheeger-Simons differential characters $[9,10,11]$ whose apparent relation with the smooth versionsof Deligne cohomology [12] and Deligne-Beilinson cohomology [13,14] developed a decade later was noticed in the early ninenties and has been reconsidered recently [15].

Virtually all the above studies deal with absolute cohomology and differential characters. A generalization of the formalism appropriate for relative cohomology and differential characters has not been fully worked out to the best of our knowledge. This is attempted in the present paper.

The reason why this is an interesting problem and not a mere academic exercise is shown by the physical examples illustrated below in which the relevance of relative cohomology and differential characters should be apparent. Since we have physical applications in mind, we want to provide a constructive treatment, i. e. one computable at least in principle. For this reason, we opt for a formulation closer in spirit to Cheeger's and Simons', which is somewhat more concrete and thus more suitable for the physicists' computational needs. We shall do this using the machinery of Čech (co)homology as in $[1,2,3]$. We shall not use partitions of unity as in [8], since these are required by distribution valued quantized fields, while the fields relevant in our examples are background semiclassical fields. Though we work mostly in the framework of relative integer cohomology, our formulation presumably might be extended to more general relative cohomology theories, in particular K-theory.

Consider a spacetime $X$ and a D-brane occupying a submanifold $Y \subseteq X$ in type II string theory. The background is characterized by the NS NS field $B_{2}$. Further, the 
D-brane carries a $U(1)$ gauge field $A_{1}$. For a string with world sheet $\Sigma_{2} \subseteq X$ such that $\partial \Sigma_{2} \subseteq Y$, the path integral measure contains a factor

$$
\operatorname{pfaff}\left(D_{\Sigma}\right) \exp \left(i \int_{\Sigma_{2}} B_{2}-i \int_{\partial \Sigma_{2}} A_{1}\right),
$$

where $D_{\Sigma}$ is the Dirac operator on $\Sigma_{2}$ and pfaff $\left(D_{\Sigma}\right)$ is its pfaffian [16]. If $\partial \Sigma_{2}=\emptyset$, the sign of pfaff $\left(D_{\Sigma}\right)$ is uniquely defined. In order for the path integral measure to be well-defined $H_{3}=d B_{2}$ is required to be a globally defined closed 3-form with quantized fluxes through any closed 3 -folds $P_{3} \subseteq X$ :

$$
\int_{P_{3}} H_{3} \in 2 \pi \mathbb{Z} .
$$

If $\partial \Sigma_{2} \neq \emptyset$, the sign of pfaff $\left(D_{\Sigma}\right)$ is not uniquely defined in general, signaling a global world-sheet anomaly. Consistency requires that this anomaly be canceled by an equal and opposite anomaly of the exponential factor of (0.1). In order for this to be possible, the 2-form $B_{A 2}=B_{2}-d A_{1}$ must be globally defined on $Y$ so that the restriction of $H_{3}$ on $Y$ is exact. The quantization condition (0.2) gets generalized as

$$
\int_{P_{3}} H_{3}-\int_{Q_{2}} B_{A 2}-\pi \int_{Q_{2}} w_{2} \in 2 \pi \mathbb{Z},
$$

for $P_{3} \subseteq X, Q_{2} \subseteq Y$ with $\partial P_{3}=Q_{2}$, where $w_{2}$ is a closed 2-form on $Y$ representing the second Stiefel-Whitney class of $Y$ modulo 2. In the simple case where $B_{2}=0, Y$ turns out to be a $\operatorname{Spin}_{c}$ manifold and $A_{1}$ a $\operatorname{Spin}_{c}$ connection. See [16] for more details and [17] for a related analysis.

The problem of $\mathrm{D}$-branes in group manifolds has received a great deal of attention recently $[18,19,20,21,22,23]$. The central issue here is the proper definition of D0 charge and its quantization. Consider a D-brane located in a submanifold $K$ of a compact simple Lie group $G$. The background is characterized by a closed 3-form $H_{3}$ on $G$, the trace of the third wedge power of the left invariant Maurer-Cartan form of $G$. According to [24], the D0 charge $Q$ of a D2-brane contained in the D-brane is defined if $H_{3}=d L_{2}$ on $K$ for some 2 -form $L_{2}$ globally defined on $K$ and is given by

$$
Q=\int_{V_{3}} H_{3}-\int_{Z_{2}} L_{2},
$$

for $V_{3} \subseteq G, Z_{2} \subseteq K$ with $\partial V_{3}=Z_{2}$. When $H_{3}$ is cohomologically trivial, $Q$ is quantized as $Q \in 2 \pi \mathbb{Z}$ in the usual way. When $H_{3}$ has a fundamental period (level) $k, Q$ is quantized as $Q \in 2 \pi \mathbb{Z}_{k}$. These quantization rules have to be compared with (0.3). 
Consider $N$ coinciding D-branes of type II string theory spanning a world-volume $W$ in the space time $X$. The background fields are the spin connection $\omega_{1}$, the NS NS B-field $B_{2}$ and the R R field $C$. Further, the set of branes carries a $U(N)$ Chan-Paton gauge field $A_{1}$ [25]. Here, we assume that $B_{2}=0 . C$ is an odd/even degree form field for type IIA/IIB strings. $C$ is not globally defined in $X$ in general. Only its field strength $G=d C$ is. The $\mathrm{D}$-brane is carries $\mathrm{R} \mathrm{R}$ charges and thus couples to the $\mathrm{R} \mathrm{R}$ field $C$ via the Wess-Zumino term. Thus, the path integral contains a factor of the form

$$
\operatorname{pfaff}\left(D_{W}\right) \exp \left(i \int_{W} \operatorname{tr} \exp _{\wedge}\left(i F_{2} / 2 \pi\right) \wedge \widehat{A}^{\frac{1}{2}}\left(R_{T W 2}\right) \wedge \widehat{A}^{-\frac{1}{2}}\left(R_{N W 2}\right) \wedge C\right)
$$

where pfaff $\left(D_{W}\right)$ is the pfaffian of the Dirac operator on $W$ and $R_{T W 2}, R_{N W 2}$ and $F_{2}$ are the curvatures of the tangent and normal bundles $T W, N W$ of $W$ and the gauge field strength, respectively. $\widehat{A}$ denotes the A-roof genus. This factor is required and explicitly determined by gauge and gravitational anomaly cancellation [26,27,28]. As before, the sign of pfaff $\left(D_{W}\right)$ suffers in general an ambiguity which signals a global anomaly. The proper definition of the path integral measure requires some kind of quantization condition for the $\mathrm{R} \mathrm{R}$ curvature $G$. This reads

$$
\int_{U} \widehat{A}^{\frac{1}{2}}\left(R_{T U 2}\right) \wedge \widehat{A}^{-\frac{1}{2}}\left(R_{N U 2}\right) \wedge G-\pi \int_{U} \nu \in 2 \pi \mathbb{Z}
$$

for any closed submanifold $U$ of $X$ of dimension one unit larger than $W$, where $R_{T W 2}$, $R_{N W 2}$ are the curvatures of the tangent and normal bundles $T U, N U$ of $U$ nd $\nu$ is a closed form representing the pfaffian anomaly modulo 2. In the last three years it has become clear that a realistic theory of $\mathrm{D}$-brane $\mathrm{R} R$ charges and $\mathrm{R} R$ fields in type II string theory requires $\mathrm{K}$ theory when $B_{2}=0$ and some sort of twisted generalization thereof when $B_{2} \neq 0[28,29,30,31,32]$. In any case, a form of generalized cohomology is involved which maps to a full lattice in ordinary real cohomology as is apparent from (0.6).

A generalization for open membranes is still to be worked out [33]. It presumably involves adding in the exponential in the right hand side of (0.5) a suitable integral on $Z=\partial Y$ leading to a structure similar to (0.1). This is however just a speculation for the time being.

The above examples show clearly that the geometrical framework suitable for the analysis of these matters is provided by relative singular homology and (some generalization of) integral cohomology. To make this clearer and also to render the rest of the paper more 
easily readable, we recall briefly some of the basic definitions. (See ref. [34,35,36,37,38,39] for background material.)

Let $X, Y$ be smooth manifolds with $Y \subseteq X$. Denote by $i: Y \rightarrow X$ the smooth inclusion map. A relative singular $p-1$-cycle $\left(S_{p-1}, T_{p-2}\right)$ of $X \bmod Y$ is a pair of singular chains of $X, Y$, respectively, satisfying

$$
\partial S_{p-1}-i_{*} T_{p-2}=0, \quad-\partial T_{p-2}=0 .
$$

A relative de Rham $p$-cocycle $\left(\Xi_{p}, \Upsilon_{p-1}\right)$ of $X \bmod Y$ is a pair of forms of $X, Y$, respectively, satisfying

$$
d \Xi_{p}=0, \quad i^{*} \Xi_{p}-d \Upsilon_{p-1}=0 .
$$

Locally, there are forms $\tilde{\Xi}_{p-1}, \tilde{\Upsilon}_{p-2}$ in $X, Y$, respectively, such that

$$
\Xi_{p}=d \tilde{\Xi}_{p-1}, \quad \Upsilon_{p-1}=i^{*} \tilde{\Xi}_{p-1}-d \tilde{\Upsilon}_{p-2}
$$

The associated relative topological integral is the formal integral

$$
\int_{S_{p-1}} \tilde{\Xi}_{p-1}-\int_{T_{p-2}} \tilde{\Upsilon}_{p-2}
$$

In general, its value is determined only up to a quantized ambiguity. In the simplest case, the ambiguity is just integer valued ${ }^{1}$. This translates into a quantization condition for the relative de Rham $p$-cocycle $\left(\Xi_{p}, \Upsilon_{p-1}\right)$ of the form

$$
\int_{s_{p}} \Xi_{p}-\int_{t_{p-1}} \Upsilon_{p-1} \in \mathbb{Z}
$$

for any relative singular $p$-cycle $\left(s_{p}, t_{p-1}\right)$. For more general quantized ambiguities, we have totally analogous generalized quantization conditions.

In the first example illustrated above, $\left(\Sigma_{2}, \partial \Sigma_{2}\right)$ is a relative singular $2-$ cycle and $\left(H_{3}, B_{A 2}\right)$ is a relative 3 -cocycle. The argument of the exponential in $(0.1)$ is the associated topological integral. The quantization condition (0.3) holds for every relative singular $3-$ cycle $\left(P_{3}, Q_{2}\right)$. Similarly, in the second example, $\left(V_{3}, Z_{2}\right)$ is a relative singular 3-cycle, $\left(H_{3}, L_{2}\right)$ is a relative 3 -cocycle and $Q$ expresses the canonical pairing of relative singular

${ }^{1}$ Here and in the following, we neglect an inessential factor $2 \pi$ appearing in the physical quantization conditions. 
3 homology and relative de Rham 3 cohomology. Quantization selects a sublattice of the latter. Similar considerations might apply to an open membrane generalization of the third example.

Since $\tilde{\Xi}_{p-1}, \tilde{\Upsilon}_{p-2}$ are only local forms in general, the proper definition of the topological integral (0.10) is not a straightforward matter. However, any reasonable definition should satisfy the following a priori requirements up to the usual quantized ambiguity. To begin with, we expect the topological integral to depend linearly on the relative cycle $\left(S_{p-1}, T_{p-2}\right)$ and the relative cocycle $\left(\Xi_{p}, \Upsilon_{p-1}\right)$. Further, we expect some kind of Stokes' theorem to hold. So, when the relative singular $p-1$-cycle $\left(S_{p-1}, T_{p-2}\right)$ is a relative boundary,

$$
S_{p-1}=\partial s_{p}-i_{*} t_{p-1}, \quad T_{p-2}=-\partial t_{p-1}
$$

for some singular chains $s_{p}, t_{p-1}$ in $X, Y$, respectively, then

$$
\int_{S_{p-1}} \tilde{\Xi}_{p-1}-\int_{T_{p-2}} \tilde{\Upsilon}_{p-2}=\int_{s_{p}} \Xi_{p}-\int_{t_{p-1}} \Upsilon_{p-1},
$$

where the integrals in the right hand side are computed according to the ordinary differential geometric prescription. Finally, we would like the topological integral to reduce to an ordinary integral when the forms $\tilde{\Xi}_{p-1}, \tilde{\Upsilon}_{p-2}$ are globally defined in $X, Y$, respectively. So, when the relative de Rham $p$-cocycle $\left(\Xi_{p}, \Upsilon_{p-1}\right)$ is a relative coboundary,

$$
\Xi_{p}=d \xi_{p-1}, \quad \Upsilon_{p-1}=i^{*} \xi_{p-1}-d v_{p-2},
$$

for some globally defined forms $\xi_{p-1}, v_{p-2}$ on $X, Y$, respectively, then

$$
\int_{S_{p-1}} \tilde{\Xi}_{p-1}-\int_{T_{p-2}} \tilde{\Upsilon}_{p-2}=\int_{S_{p-1}} \xi_{p-1}-\int_{T_{p-2}} v_{p-2}
$$

where again the integrals in the right hand side are computed according to the ordinary differential geometric prescription.

The plan of the paper is as follows. In sect. 1, we introduce the basic notions of relative homology and cohomology. In sect. 2, we provide an explicit construction of the family of relative Cheeger-Simons differential characters and show independence form covering choices. In sect. 3, we analyze in detail its formal properties. Finally, sect. 4 contains a few concluding remarks. 


\section{Relative singular, de Rham and Čech (co)homology}

This is a review of some basic material on relative singular, de Rham and Cech (co)homology. The reader interested in a more thorough treatment is suggested to consult standard textbooks such as $[34,35,36]$.

\section{Basic definitions and facts}

Let $M$ be a smooth manifold. Let $\mathcal{O}=\left\{O_{\alpha} \mid \alpha \in A\right\}$ be an open covering of $M$. Here, $A$ is a countable index set. We set, for $k \geq 0$,

$$
O_{\alpha_{0}, \ldots, \alpha_{k}}=O_{\alpha_{0}} \cap \cdots \cap O_{\alpha_{k}}
$$

The $k$-th nerve of $\mathcal{O}$ is

$$
N(\mathcal{O}, k)=\left\{\left(\alpha_{0}, \ldots, \alpha_{k}\right) \in A^{k+1} \mid O_{\alpha_{0}, \ldots, \alpha_{k}} \neq \emptyset\right\} .
$$

$\mathcal{O}$ is a good covering if all the non empty $O_{\alpha_{0}, \ldots, \alpha_{k}}$ are contractible.

For $r \in \mathbb{Z}$, we denote by $\mathcal{S}_{r}(M)$ the group of (generalized) dimension $r$ singular chains of $M: \mathcal{S}_{r}(M)=0$, for $r \leq-2, \mathcal{S}_{-1}(M)=\mathbb{Z}$ and $\mathcal{S}_{r}(M)$ is the group of ordinary smooth, finite singular chains of $M$ of dimension $r$, for $0 \leq r$. A dimension $r$ singular chain $U_{r}$ is characterized by its support $\operatorname{supp} U_{r} \subseteq M$. By convention, supp $U_{r}=\emptyset$ for $r \leq-1$. For any non empty open subset $O$ of $M$, we denote by $\mathcal{S}_{r}^{O}(M)$ the group of all dimension $r$ chains $U_{r}$ such that $\operatorname{supp} U_{r} \subseteq O$. Clearly, $\mathcal{S}_{r}^{O}(M)$ is a subgroup of $\mathcal{S}_{r}(M)$.

We define a homomorphism $b: \mathcal{S}_{r}(M) \rightarrow \mathcal{S}_{r-1}(M)$ by

$$
b U_{r}=\partial_{s} U_{r} .
$$

Here, for $1 \leq r, \partial_{s}$ is the customary simplicial boundary operator, while, for $r=0$, $\partial_{s} U_{0}=\operatorname{ind} U_{0}$, where ind $\sum_{P} n_{P} P=\sum_{P} n_{P}$ for a dimension 0 chain $\sum_{P} n_{P} P{ }^{1} . b$ is nilpotent

$$
b^{2}=0
$$

${ }^{1}$ In dimension 0 , the definition of the boundary operator $b$ given here differs from the customary one of singular homology, where $b$ vanishes. As a consequence, the 0 dimensional homology groups corresponding to the two definitions of $b$ are also different. Our definition ensures that the statement above (1.1.7) holds. 
Let $\mathcal{O}$ be an open covering of $M$. For $r \in \mathbb{Z}$, we denote by $\mathcal{S}_{r}^{\mathcal{O}}(M)$ the subgroup of $\mathcal{O}$-small elements of $\mathcal{S}_{r}(M): \mathcal{S}_{r}^{\mathcal{O}}(M)=\mathcal{S}_{r}(M)$, for $r \leq 0$, and $\mathcal{S}_{r}^{\mathcal{O}}(M)$ is the subgroup of $\mathcal{S}_{r}(M)$ formed by the singular chains made up of simplices the support of each of which is contained in some open set of $\mathcal{O}$, for $1 \leq r$. There exists a homomorphism $q: \mathcal{S}_{r}(M) \rightarrow \mathcal{S}_{r}(M)$, called barycentric subdivision operator, with the following properties. $q$ is a chain map

$$
q b-b q=0 .
$$

$q$ is homotopic to the identity, i. e. there is a homomorphism $c: \mathcal{S}_{r}(M) \rightarrow \mathcal{S}_{r+1}(M)$ such that

$$
b c+c b=q-1 .
$$

Most importantly, for any $U_{r} \in \mathcal{S}_{r}(M)$ there is an integer $k\left(U_{r}, \mathcal{O}\right) \geq 0$ such that $q^{k} U_{r} \in$ $\mathcal{S}_{r}^{\mathcal{O}}(M)$ for $k \geq k\left(U_{r}, \mathcal{O}\right)$. $q$ and $c$ preserve $\mathcal{O}$-smallness: for any $U_{r} \in \mathcal{S}_{r}^{\mathcal{O}}(M), q U_{r} \in$ $\mathcal{S}_{r}^{\mathcal{O}}(M)$ and $c U_{r} \in \mathcal{S}_{r+1}^{\mathcal{O}}(M)$. Further, for any $U_{r} \in \mathcal{S}_{r}(M), c U_{r}$ is degenerate, i. e. it is made up of simplices each of which, considered as a smooth map of the standard $r+1$ simplex into $M$, has rank smaller than $r+1$. An explicit construction of $q$ and $c$ can be found in [36].

Let $\mathcal{O}=\left\{O_{\alpha} \mid \alpha \in A\right\}$ be an open covering of $M$. For $k, r \in \mathbb{Z}$, we denote by $\mathcal{C}_{k, r}(M, \mathcal{O})$ the group of finite Čech singular chains of $\mathcal{O}$ in $M$ of Čech degree $k$ and dimension $r$ : $\mathcal{C}_{k, r}(M, \mathcal{O})=0$, for $k \leq-2, \mathcal{C}_{-1, r}(M, \mathcal{O})=\mathcal{S}_{r}^{\mathcal{O}}(M)$ and $\mathcal{C}_{k, r}(M, \mathcal{O})$ is the group of alternating maps $U_{k, r}: A^{k+1} \rightarrow \mathcal{S}_{r}^{\mathcal{O}}(M)$ such that $\left(U_{k, r}\right)_{\alpha_{0}, \ldots, \alpha_{k}}=0$ for $\left(\alpha_{0}, \ldots, \alpha_{k}\right) \notin$ $N(\mathcal{O}, k),\left(U_{k, r}\right)_{\alpha_{0}, \ldots, \alpha_{k}} \in \mathcal{S}_{r}^{O_{\alpha_{0}, \ldots, \alpha_{k}}}(M)$ for $\left(\alpha_{0}, \ldots, \alpha_{k}\right) \in N(\mathcal{O}, k)$ and $\left(U_{k, r}\right)_{\alpha_{0}, \ldots, \alpha_{k}} \neq 0$ only for a finite number of $\left(\alpha_{0}, \ldots, \alpha_{k}\right)$, for $0 \leq k$. Note that the Čech singular chains are automatically $\mathcal{O}$-small. The Cech singular chains of $\mathcal{C}_{-1, r}(M, \mathcal{O})$ are called simply singular chains, on account of the definition given above. The Čech singular chains of $\mathcal{C}_{k,-1}(M, \mathcal{O})$ are called integer Čech chains, since they are integer valued.

The operator $b$ yields a homomorphism $b: \mathcal{C}_{k, r}(M, \mathcal{O}) \rightarrow \mathcal{C}_{k, r-1}(M, \mathcal{O})$ in obvious fashion. It is known that the homology of $\left(\mathcal{C}_{k, *}(M, \mathcal{O}), b\right)$ vanishes for $k>-1$, if $\mathcal{O}$ is a good covering.

We define a homomorphism $\beta: \mathcal{C}_{k, r}(M, \mathcal{O}) \rightarrow \mathcal{C}_{k-1, r}(M, \mathcal{O})$ by

$$
\left(\beta U_{k, r}\right)_{\alpha_{0}, \ldots, \alpha_{k-1}}=\sum_{\alpha \in A}\left(U_{k, r}\right)_{\alpha, \alpha_{0}, \ldots, \alpha_{k-1}} .
$$

$\beta$ is a differential

$$
\beta^{2}=0
$$


The homology of $\left(\mathcal{C}_{*, r}(M, \mathcal{O}), \beta\right)$ is known to vanish for $r>-1$ for any covering $\mathcal{O}$.

$b$ and $\beta$ commute

$$
b \beta-\beta b=0 .
$$

For $r \in \mathbb{Z}$, we denote by $\mathcal{D}^{r}(M)$ the vector space of (generalized) degree $r$ differential forms of $M: \mathcal{D}^{r}(M)=0$, for $r \leq-2, \mathcal{D}^{-1}(M)=\mathbb{R}$ and $\mathcal{D}^{r}(M)$ is the vector space of ordinary smooth differential forms of $M$ of de Rham degree $r$, for $0 \leq r$. More generally, one may consider degree $r$ differential forms $\Xi^{r}$ which are defined only on a domain $\operatorname{dom} \Xi^{r} \subseteq$ $M$. By convention, dom $\Xi^{r}=M$ for $r \leq-1$. For any non empty open subset $O$ of $M$, we denote by $\mathcal{D}_{O}^{r}(M)$ the vector space of all degree $r$ forms $\Xi^{r}$ such that dom $\Xi^{r} \supseteq O$. Clearly, $\mathcal{D}^{r}(M)$ is a subspace of $\mathcal{D}_{O}^{r}(M)$.

We define a homomorphism $d: \mathcal{D}^{r}(M) \rightarrow \mathcal{D}^{r+1}(M)$ by

$$
d \Xi^{r}=d_{d R} \Xi^{r}
$$

Here, for $0 \leq r, d_{d R}$ is the usual de Rham differential while, for $r=-1, d_{d R} \Xi^{-1}$ is the constant 0 -form corresponding to the constant $\Xi^{-1}{ }^{2} . d$ is a differential

$$
d^{2}=0
$$

Let $\mathcal{O}=\left\{O_{\alpha} \mid \alpha \in A\right\}$ be an open covering of $M$. For $k, r \in \mathbb{Z}$, we denote by $\mathcal{C}^{k, r}(M, \mathcal{O})$ the vector space of Čech-de Rham cochains of $\mathcal{O}$ in $M$ of Čech degree $k$ and de Rham degree $r: \mathcal{C}^{k, r}(M, \mathcal{O})=0$, for $k \leq-2, \mathcal{C}^{-1, r}(M, \mathcal{O})$ is the vector space of forms $\Xi^{r} \in$ $\mathcal{D}^{r}(M)$ and $\mathcal{C}^{k, r}(M, \mathcal{O})$ is the vector space of alternating maps $\Xi^{k, r}: A^{k+1} \rightarrow \mathcal{D}^{r}(M)$ such that $\left(\Xi^{k, r}\right)_{\alpha_{0}, \ldots, \alpha_{k}}=0$ for $\left(\alpha_{0}, \ldots, \alpha_{k}\right) \notin N(\mathcal{O}, k)$ and $\left(\Xi^{k, r}\right)_{\alpha_{0}, \ldots, \alpha_{k}} \in \mathcal{D}_{O_{\alpha_{0}, \ldots, \alpha_{k}}}^{r}(M)$ for $\left(\alpha_{0}, \ldots, \alpha_{k}\right) \in N(\mathcal{O}, k)$, for $0 \leq k$. The Čech-de Rham cochains of $\mathcal{C}^{-1, r}(M, \mathcal{O})$ are called simply de Rham cochains, on account of the definition given above. The Čech-de Rham cochains of $\mathcal{C}^{k,-1}(M, \mathcal{O})$ are called real Čech cochains, since they are real valued.

The operator $d$ yields a homomorphism $d: \mathcal{C}^{k, r}(M, \mathcal{O}) \rightarrow \mathcal{C}^{k, r+1}(M, \mathcal{O})$. By Poincaré's lemma, the cohomology of $\left(\mathcal{C}^{k, *}(M, \mathcal{O}), d\right)$ vanishes for $k>-1$, if $\mathcal{O}$ is a good covering.

2 The definition given here of coboundary operator $d$ in degree -1 is a rather natural extension of the usual de Rham differential which allows the treatment of degree -1 on the same footing as non negative degree. It further ensures that the statement above (1.1.12) holds. 
We define a homomorphism $\delta: \mathcal{C}^{k, r}(M, \mathcal{O}) \rightarrow \mathcal{C}^{k+1, r}(M, \mathcal{O})$ by

$$
\left(\delta \Xi^{k, r}\right)_{\alpha_{0}, \ldots, \alpha_{k+1}}=\left.\sum_{l=0}^{k+1}(-1)^{l}\left(\Xi^{k, r}\right)_{\alpha_{0}, \ldots, \alpha_{l-1}, \alpha_{l+1}, \ldots, \alpha_{k+1}}\right|_{O_{\alpha_{0}, \ldots, \alpha_{k+1}}}
$$

$\delta$ is nilpotent

$$
\delta^{2}=0
$$

The cohomology of $\left(\mathcal{C}^{*, r}(M, \mathcal{O}), \delta\right)$ is known to vanish for $r>-1$ for any covering $\mathcal{O}$.

$d$ and $\delta$ commute

$$
d \delta-\delta d=0
$$

A degree $k$ real Čech cochain $\Xi^{k,-1} \in \mathcal{C}^{k,-1}(M, \mathcal{O})$ is called integer if $\left(\Xi^{k,-1}\right)_{\alpha_{0}, \ldots, \alpha_{k}} \in$ $\mathbb{Z}$ for all $\left(\alpha_{0}, \ldots, \alpha_{k}\right) \in A^{k+1}$. Such integer cochains form a lattice subgroup $\mathcal{C}_{\mathbb{Z}}^{k,-1}(M, \mathcal{O})$ of $\mathcal{C}^{k,-1}(M, \mathcal{O})$.

Let $U_{k, r} \in \mathcal{C}_{k, r}(M, \mathcal{O}), \Xi^{k, r} \in \mathcal{C}^{k, r}(M, \mathcal{O})$. For $k \geq 0$, we set

$$
\left\langle U_{k, r}, \Xi^{k, r}\right\rangle= \begin{cases}\frac{1}{k !} \sum_{\left(\alpha_{0}, \ldots, \alpha_{k}\right) \in N(\mathcal{O}, k)} \int_{\left(U_{k, r}\right)_{\alpha_{0}, \ldots, \alpha_{k}}}\left(\Xi^{k, r}\right)_{\alpha_{0}, \ldots, \alpha_{k}}, & \text { if } r \geq 0, \\ \frac{1}{k !} \sum_{\left(\alpha_{0}, \ldots, \alpha_{k}\right) \in N(\mathcal{O}, k)}\left(U_{k,-1}\right)_{\alpha_{0}, \ldots, \alpha_{k}}\left(\Xi^{k,-1}\right)_{\alpha_{0}, \ldots, \alpha_{k}}, & \text { if } r=-1, \\ 0, & \text { if } r \leq-2 .\end{cases}
$$

For $k=-1$, similar expressions hold but with the sum over the $k$-th nerve of the covering and the factor $1 / k$ ! omitted. The integrals in the right hand side are convergent, since all singular chains have compact support. The sum in the right hand side is convergent, as all Čech singular chains are finite by definition. One has

$$
\begin{aligned}
& \left\langle U_{k, r}, d \Xi^{k, r-1}\right\rangle=\left\langle b U_{k, r}, \Xi^{k, r-1}\right\rangle, \\
& \left\langle U_{k, r}, \delta \Xi^{k-1, r}\right\rangle=\left\langle\beta U_{k, r}, \Xi^{k-1, r}\right\rangle .
\end{aligned}
$$

These duality relations play a fundamental role in the following.

Let $\mathcal{O}=\left\{O_{\alpha} \mid \alpha \in A\right\}, \mathcal{O}^{\prime}=\left\{O^{\prime}{ }_{\alpha^{\prime}} \mid \alpha^{\prime} \in A^{\prime}\right\}$ be open coverings of the manifold M. $\mathcal{O}^{\prime}$ is called a refinement of $\mathcal{O}$ if there is a map $f: A^{\prime} \rightarrow A$ such that $O^{\prime}{ }_{\alpha^{\prime}} \subseteq O_{f\left(\alpha^{\prime}\right)}$ for $\alpha^{\prime} \in A^{\prime}$. The refinement map $f$ defines a homomorphism $f^{*}: \mathcal{C}^{k, r}(M, \mathcal{O}) \rightarrow \mathcal{C}^{k, r}\left(M, \mathcal{O}^{\prime}\right)$ of the corresponding spaces of Čech-de Rham cochains by

$$
f^{*} \Xi^{k, r}{ }_{\alpha^{\prime}{ }_{0} \ldots \alpha^{\prime}{ }_{l}}=\left.\Xi^{k, r} f\left(\alpha^{\prime}{ }_{0}\right) \ldots f\left(\alpha^{\prime}{ }_{l}\right)\right|_{O_{\alpha^{\prime}{ }_{0} \ldots \alpha^{\prime} l}} .
$$


$f^{*}$ is a cochain map, i. e.

$$
f^{*} \delta=\delta^{\prime} f^{*}
$$

The resulting homomorphism of cohomology depends only on the coverings $\mathcal{O}, \mathcal{O}^{\prime}$ but not on the refinement map $f$.

In the rest of this section, we shall describe briefly the main versions of $Y$ relative homology and cohomology of $X$ for a pair of manifolds $X, Y$ such that $Y \subseteq X$.

Let $p \in \mathbb{N}, p \geq 2$. Let $X, Y$ be smooth manifolds with $\operatorname{dim} X \geq p, \operatorname{dim} Y \geq p-1$ and such that $Y \subseteq X$. Let $i: Y \rightarrow X$ be the smooth inclusion map.

Let $\mathcal{O}$ be an open covering of $X$ and let $\mathcal{O} \cap Y$ be the open covering of $Y$ induced by $\mathcal{O}$.

\section{Relative homology and cohomology}

A $Y$ relative singular $p-1$-chain $\left(S_{p-1}, T_{p-2}\right)$ of $X$ is a pair of singular chains $S_{p-1} \in$ $\mathcal{S}_{p-1}(X), T_{p-2} \in \mathcal{S}_{p-2}(Y)$. A $Y$ relative singular $p-1$-chain $\left(S_{p-1}, T_{p-2}\right)$ of $X$ is a cycle if

$$
\begin{aligned}
& b S_{p-1}-i_{*} T_{p-2}=0, \\
& -b T_{p-2}=0 .
\end{aligned}
$$

A $Y$ relative singular $p-1$-cycle $\left(S_{p-1}, T_{p-2}\right)$ of $X$ is a boundary if it is of the form

$$
\begin{aligned}
& S_{p-1}=b s_{p}-i_{*} t_{p-1}, \\
& T_{p-2}=-b t_{p-1},
\end{aligned}
$$

where $\left(s_{p}, t_{p-1}\right)$ is an arbitrary $Y$ relative singular $p$-chain of $X$. We denote by $C_{p-1}^{s}(X, Y)$, $Z_{p-1}^{s}(X, Y), B_{p-1}^{s}(X, Y)$ the groups of $Y$ relative singular $p-1$-chains, cycles and boundaries of $X$, respectively. Two relative $p-1$-cycles are equivalent if their difference is a relative $p-1$-boundary. The equivalence classes of $Y$ relative singular $p-1$-cycles of $X$ form the $p$-1-th relative singular homology group $H_{p-1}^{s}(X, Y)$.

A $Y$ relative singular $p-1$-chain (respectively a cycle, a boundary) $\left(S_{p-1}, T_{p-2}\right)$ is said $\mathcal{O}$-small if $S_{p-1}$ is $\mathcal{O}$-small and $T_{p-2}$ is $\mathcal{O} \cap Y$-small in the sense defined in the previous subsection. We denote by $C_{p-1}^{s \mathcal{O}}(X, Y), Z_{p-1}^{s \mathcal{O}}(X, Y), B_{p-1}^{s \mathcal{O}}(X, Y)$ the groups of $\mathcal{O}$-small 
$Y$ relative singular $p-1$-chains, cycles and boundaries of $X$, respectively. Two $\mathcal{O}$-small relative $p-1$-cycles are equivalent if their difference is an $\mathcal{O}$-small relative $p-1$-boundary. The equivalence classes of $\mathcal{O}$-small $Y$ relative singular $p-1$-cycles of $X$ form the $p-1$-th $\mathcal{O}$-small relative singular homology group $H_{p-1}^{s \mathcal{O}}(X, Y)$.

An $\mathcal{O}$-small $Y$ relative singular $p-1$-chain $\left(S_{p-1}, T_{p-2}\right)$ can be viewed as a pair of Čech singular chains $\left(S_{-1, p-1}, T_{-1, p-2}\right)$ with $S_{-1, p-1} \in \mathcal{C}_{-1, p-1}(X, \mathcal{O}), T_{-1, p-2} \in \mathcal{C}_{-1, p-2}(Y, \mathcal{O} \cap$ $Y)$. We shall use both notations interchangeably depending on context.

A $Y$ relative integer Čech $p-1$-chain $\left(S_{p-1,-1}, T_{p-2,-1}\right)$ of $X$ is a pair of integer Čech chains $S_{p-1,-1} \in \mathcal{C}_{p-1,-1}(X, \mathcal{O}), T_{p-2,-1} \in \mathcal{C}_{p-2,-1}(Y, \mathcal{O} \cap Y)$. A $Y$ relative integer Cech $p-1$-chain $\left(S_{p-1,-1}, T_{p-2,-1}\right)$ of $X$ is a cycle if

$$
\begin{aligned}
& \beta S_{p-1,-1}-i_{*} T_{p-2,-1}=0 \\
& -\beta T_{p-2,-1}=0 .
\end{aligned}
$$

A $Y$ relative integer Čech $p-1$-cycle $\left(S_{p-1,-1}, T_{p-2,-1}\right)$ of $X$ is a boundary if it is of the form

$$
\begin{aligned}
& S_{p-1,-1}=\beta s_{p,-1}-i_{*} t_{p-1,-1}, \\
& T_{p-2,-1}=-\beta t_{p-1,-1}
\end{aligned}
$$

where $\left(s_{p,-1}, t_{p-1,-1}\right)$ is an arbitrary $Y$ relative integer Čech $p$-chain of $X$. We denote by $C_{p-1}^{C}(X, Y, \mathcal{O}), Z_{p-1}^{C}(X, Y, \mathcal{O}), B_{p-1}^{C}(X, Y, \mathcal{O})$ the groups of $Y$ relative integer Cech $p-1$-chains, cycles and boundaries of $X$, respectively. Two relative $p-1$-cycles are equivalent if their difference is a relative $p-1$-boundary. The equivalence classes of $Y$ relative integer Čech $p-1-$ cycles of $X$ form the $p-1-$ th relative integer Čech homology group $H_{p-1}^{C}(X, Y, \mathcal{O})$.

For $r \in \mathbb{N}$, set $I_{r}=\{0,1,2, \ldots, r\}$. A $Y$ relative Čech singular $p-1$-intertwiner of $X$ is a sequence $\left(S_{-1, p-1}, T_{-1, p-2} ;\left\{V_{k, p-1-k} \mid k \in I_{p-1}\right\},\left\{Z_{k, p-2-k} \mid k \in I_{p-2}\right\} ; S_{p-1,-1}, T_{p-2,-1}\right)$ with $S_{-1, p-1} \in \mathcal{C}_{-1, p-1}(X, \mathcal{O}), T_{-1, p-2} \in \mathcal{C}_{-1, p-2}(Y, \mathcal{O} \cap Y), V_{k, p-1-k} \in \mathcal{C}_{k, p-1-k}(X, \mathcal{O})$, $Z_{k, p-2-k} \in \mathcal{C}_{k, p-2-k}(Y, \mathcal{O} \cap Y), S_{p-1,-1} \in \mathcal{C}_{p-1,-1}(X, \mathcal{O}), T_{p-2,-1} \in \mathcal{C}_{p-2,-1}(Y, \mathcal{O} \cap Y)$ satisfying

$$
S_{-1, p-1}=\beta V_{0, p-1},
$$




$$
\begin{aligned}
& T_{-1, p-2}=\beta Z_{0, p-2}, \\
& b V_{k, p-1-k}=\beta V_{k+1, p-2-k}+(-1)^{k} i_{*} Z_{k, p-2-k}, \quad 0 \leq k \leq p-2, \\
& b Z_{k, p-2-k}=\beta Z_{k+1, p-3-k}, \quad 0 \leq k \leq p-3, \\
& S_{p-1,-1}=b V_{p-1,0}, \\
& T_{p-2,-1}=-(-1)^{p-2} b Z_{p-2,0} .
\end{aligned}
$$

Note that $\left(S_{p-1}, T_{p-2}\right) \in Z_{p-1}^{s \mathcal{O}}(X, Y)$ (cfr. eq. (1.2.1)) and $\left(S_{p-1,-1}, T_{p-2,-1}\right) \in Z_{p-1}^{C}(X$, $Y, \mathcal{O})\left(\right.$ cfr. eq. (1.2.3)). A $Y$ relative singular Čech $p-1$-intertwiner $\left(S_{-1, p-1}, T_{-1, p-2}\right.$; $\left.\left\{V_{k, p-1-k} \mid k \in I_{p-1}\right\},\left\{Z_{k, p-2-k} \mid k \in I_{p-2}\right\} ; S_{p-1,-1}, T_{p-2,-1}\right)$ of $X$ is said trivial if

$$
\begin{aligned}
& S_{-1, p-1}=b s_{-1, p}-i_{*} t_{-1, p-1} \\
& T_{-1, p-2}=-b t_{-1, p-1} \\
& V_{k, p-1-k}=b v_{k, p-k}+\beta v_{k+1, p-1-k}+(-1)^{k} i_{*} z_{k, p-1-k}, \quad 0 \leq k \leq p-1 \\
& Z_{k, p-2-k}=b z_{k, p-1-k}+\beta z_{k+1, p-2-k}, \quad 0 \leq k \leq p-2 \\
& S_{p-1,-1}=\beta s_{p,-1}-i_{*} t_{p-1,-1} \\
& T_{p-2,-1}=-\beta t_{p-1,-1}
\end{aligned}
$$

where $s_{-1, p} \in \mathcal{C}_{-1, p}(X, \mathcal{O}), t_{-1, p-1} \in \mathcal{C}_{-1, p-1}(Y, \mathcal{O} \cap Y), v_{k, p-k} \in \mathcal{C}_{k, p-k}(X, \mathcal{O})$, for $0 \leq$ $k \leq p, z_{k, p-1-k} \in \mathcal{C}_{k, p-1-k}(Y, \mathcal{O} \cap Y)$, for $0 \leq k \leq p-1, s_{p,-1} \in \mathcal{C}_{p,-1}(X, \mathcal{O}), t_{p-1,-1} \in$ $\mathcal{C}_{p-1,-1}(Y, \mathcal{O} \cap Y)$ are such that

$$
\begin{aligned}
& s_{-1, p}=\beta v_{0, p}, \\
& t_{-1, p-1}=-\beta z_{0, p-1}, \\
& s_{p,-1}=b v_{p, 0} \\
& t_{p-1,-1}=-(-1)^{p-1} b z_{p-1,0} .
\end{aligned}
$$


We denote by $Z I_{p-1}^{C s}(X, Y, \mathcal{O}), B I_{p-1}^{C s}(X, Y, \mathcal{O})$ the groups of $Y$ relative Čech singular $p-1-$ intertwiners, and trivial intertwiners of $X$, respectively. Two relative $p-1$-intertwiners are equivalent if their difference is trivial. The equivalence classes of $Y$ relative Čech singular $p-1$-intertwiners of $X$ form a group $H I_{p-1}^{C s}(X, Y, \mathcal{O})$. The notion of intertwiner given here is the generalization of that of 'element' of [39] suitable for relative homology.

A $Y$ relative de Rham $p$-cochain $\left(\Xi^{p}, \Upsilon^{p-1}\right)$ of $X$ is a pair of de Rham cochains $\Xi^{p} \in \mathcal{D}^{p}(X), \Upsilon^{p-1} \in \mathcal{D}^{p-1}(Y)$. A $Y$ relative de Rham $p$-cochain $\left(\Xi^{p}, \Upsilon^{p-1}\right)$ of $X$ is a cocycle if

$$
\begin{aligned}
& d \Xi^{p}=0, \\
& i^{*} \Xi^{p}-d \Upsilon^{p-1}=0 .
\end{aligned}
$$

A $Y$ relative de Rham $p$-cocycle $\left(\Xi^{p}, \Upsilon^{p-1}\right)$ of $X$ is a coboundary if it is of the form

$$
\begin{aligned}
& \Xi^{p}=d \xi^{p-1}, \\
& \Upsilon^{p-1}=i^{*} \xi^{p-1}-d v^{p-2},
\end{aligned}
$$

where $\left(\xi^{p-1}, v^{p-2}\right)$ is an arbitrary $Y$ relative de Rham $p-1$-cochain of $X$. We denote by $C_{d R}^{p}(X, Y), Z_{d R}^{p}(X, Y), B_{d R}^{p}(X, Y)$ the vector spaces of $Y$ relative de Rham $p$-cochains, cocycles and coboundaries of $X$, respectively. Two relative $p$-cocycles are equivalent if their difference is a $p$-coboundary. The equivalence classes of $Y$ relative de Rham $p$-cocycles of $X$ span the $p$-th relative de Rham cohomology space $H_{d R}^{p}(X, Y)$.

A $Y$ relative de Rham $p-1$-cochain $\left(\Xi^{p}, \Upsilon^{p-1}\right)$ can be viewed as a pair of Čech-de Rham cochains $\left(\Xi^{-1, p}, \Upsilon^{-1, p-1}\right)$ with $\Xi^{-1, p} \in \mathcal{C}^{-1, p}(X, \mathcal{O}), \Upsilon^{-1, p-1} \in \mathcal{C}^{-1, p-1}(Y, \mathcal{O} \cap Y)$. We shall use both notations interchangeably depending on context.

A $Y$ relative real Čech $p$-cochain $\left(\Xi^{p,-1}, \Upsilon^{p-1,-1}\right)$ of $X$ is a pair of real Čech cochains $\Xi^{p,-1} \in \mathcal{C}^{p,-1}(X, \mathcal{O}), \Upsilon^{p-1,-1} \in \mathcal{C}^{p-1,-1}(Y, \mathcal{O} \cap Y)$. A $Y$ relative real Čech $p$-cochain $\left(\Xi^{p,-1}, \Upsilon^{p-1,-1}\right)$ of $X$ is a cocycle if

$$
\begin{aligned}
& \delta \Xi^{p,-1}=0, \\
& i^{*} \Xi^{p,-1}-\delta \Upsilon^{p-1,-1}=0 .
\end{aligned}
$$


A $Y$ relative real Čech $p$-cocycle $\left(\Xi^{p,-1}, \Upsilon^{p-1,-1}\right)$ of $X$ is a coboundary if it is of the form

$$
\begin{aligned}
& \Xi^{p,-1}=\delta \xi^{p-1,-1}, \\
& \Upsilon^{p-1,-1}=i^{*} \xi^{p-1,-1}-\delta v^{p-2,-1},
\end{aligned}
$$

where $\left(\xi^{p-1,-1}, v^{p-2,-1}\right)$ is an arbitrary $Y$ relative real Čech $p-1$-cochain of $X$. We denote by $C_{C}^{p}(X, Y, \mathcal{O}), Z_{C}^{p}(X, Y, \mathcal{O}), B_{C}^{p}(X, Y, \mathcal{O})$ the vector spaces of $Y$ relative real Čech $p$-cochains, cocycles and coboundaries of $X$, respectively. Two relative $p$-cocycles are equivalent if their difference is a $p$-coboundary. The equivalence classes of $Y$ relative real Čech $p$-cocycles of $X$ form the $p$-th relative real Čech cohomology space $H_{C}^{p}(X, Y, \mathcal{O})$.

For $r \in \mathbb{N}$, set $I^{r}=\{0,1,2, \ldots, r\}$. A $Y$ relative Čech-de Rham $p$-cointertwiner of $X$ is a sequence $\left(\Xi^{-1, p}, \Upsilon^{-1, p-1} ;\left\{\Omega^{k, p-1-k} \mid k \in I^{p-1}\right\},\left\{\Theta^{k, p-2-k} \mid k \in I^{p-2}\right\} ; \Xi^{p,-1}, \Upsilon^{p-1,-1}\right)$, where $\Xi^{-1, p} \in \mathcal{C}^{-1, p}(X, \mathcal{O}), \Upsilon^{-1, p-1} \in \mathcal{C}^{-1, p-1}(Y, \mathcal{O} \cap Y), \Omega^{k, p-1-k} \in \mathcal{C}^{k, p-1-k}(X, \mathcal{O})$, $\Theta^{k, p-2-k} \in \mathcal{C}^{k, p-2-k}(Y, \mathcal{O} \cap Y), \Xi^{p,-1} \in \mathcal{C}^{p,-1}(X, \mathcal{O}), \Upsilon^{p-1,-1} \in \mathcal{C}^{p-1,-1}(Y, \mathcal{O} \cap Y)$ satisfy

$$
\begin{aligned}
& \delta \Xi^{-1, p}=d \Omega^{0, p-1} \\
& \delta \Upsilon^{-1, p-1}=-d \Theta^{0, p-2}+i^{*} \Omega^{0, p-1} \\
& d \Omega^{k, p-1-k}=\delta \Omega^{k-1, p-k}, \quad 1 \leq k \leq p-1, \\
& d \Theta^{k, p-2-k}=\delta \Theta^{k-1, p-1-k}+(-1)^{k} i^{*} \Omega^{k, p-1-k}, \quad 1 \leq k \leq p-2, \\
& d \Xi^{p,-1}=\delta \Omega^{p-1,0} \\
& d \Upsilon^{p-1,-1}=(-1)^{p-1}\left(\delta \Theta^{p-2,0}+(-1)^{p-1} i^{*} \Omega^{p-1,0}\right) .
\end{aligned}
$$

Note that $\left(\Xi^{p}, \Upsilon^{p-1}\right) \in Z_{d R}^{p}(X, Y)$ (cfr. eq. (1.2.13)) and $\left(\Xi^{p,-1}, \Upsilon^{p-1,-1}\right) \in Z_{C}^{p}(X, Y, \mathcal{O})$ (cfr. eq. (1.2.15)). We call a $Y$ relative Čech-de Rham $p$-cointertwiner $\left(\Xi^{-1, p}, \Upsilon^{-1, p-1}\right.$; $\left.\left\{\Omega^{k, p-1-k} \mid k \in I^{p-1}\right\},\left\{\Theta^{k, p-2-k} \mid k \in I^{p-2}\right\} ; \Xi^{p,-1}, \Upsilon^{p-1,-1}\right)$ of $X$ trivial if

$$
\begin{aligned}
& \Xi^{-1, p}=d \xi^{-1, p-1}, \\
& \Upsilon^{-1, p-1}=i^{*} \xi^{-1, p-1}-d v^{-1, p-2}, \\
& \Omega^{k, p-1-k}=d \omega^{k, p-2-k}+\delta \omega^{k-1, p-1-k}, \quad 0 \leq k \leq p-1,
\end{aligned}
$$




$$
\begin{aligned}
& \Theta^{k, p-2-k}=d \theta^{k, p-3-k}+\delta \theta^{k-1, p-2-k}+(-1)^{k} i^{*} \omega^{k, p-2-k}, \quad 0 \leq k \leq p-2, \\
& \Xi^{p,-1}=\delta \xi^{p-1,-1} \\
& \Upsilon^{p-1,-1}=i^{*} \xi^{p-1,-1}-\delta v^{p-2,-1},
\end{aligned}
$$

where $\xi^{-1, p-1} \in \mathcal{C}^{-1, p-1}(X, \mathcal{O}), v^{-1, p-2} \in \mathcal{C}^{-1, p-2}(Y, \mathcal{O} \cap Y), \omega^{k, p-2-k} \in \mathcal{C}^{k, p-2-k}(X, \mathcal{O})$, for $-1 \leq k \leq p-1, \theta^{k, p-3-k} \in \mathcal{C}^{k, p-3-k}(Y, \mathcal{O} \cap Y)$, for $-1 \leq k \leq p-2, \xi^{p-1,-1} \in$ $\mathcal{C}^{p-1,-1}(X, \mathcal{O}), v^{p-2,-1} \in \mathcal{C}^{p-2,-1}(Y, \mathcal{O} \cap Y)$ with

$$
\begin{aligned}
& \xi^{-1, p-1}=\omega^{-1, p-1}, \\
& v^{-1, p-2}=\theta^{-1, p-2}, \\
& \xi^{p-1,-1}=\omega^{p-1,-1}, \\
& v^{p-2,-1}=(-1)^{p-2} \theta^{p-2,-1} .
\end{aligned}
$$

We denote by $Z I_{C d R}^{p}(X, Y, \mathcal{O}), B I_{C d R}^{p}(X, Y, \mathcal{O})$ the spaces of $Y$ relative Čech-de Rham $p$ cointertwiners, and trivial cointertwiners of $X$, respectively. Two relative $p$-cointertwiners are equivalent if their difference is trivial. The equivalence classes of $Y$ relative Čech-de Rham $p$-cointertwiners of $X$ form a space $H I_{C d R}^{p}(X, Y, \mathcal{O})$. The notion of cointertwiner given here is the generalization of that of 'coelement' of [39] suitable for relative cohomology.

\section{Integral relative $\check{C} e c h$ cohomology and relative differential cocycles}

A $Y$ relative integer Čech $p$-cochain $\left(\hat{\Xi}^{p,-1}, \hat{\Upsilon}^{p-1,-1}\right)$ of $X$ is a pair of integer Čech cochains $\hat{\Xi}^{p,-1} \in \mathcal{C}_{\mathbb{Z}}^{p,-1}(X, \mathcal{O}), \hat{\Upsilon}^{p-1,-1} \in \mathcal{C}_{\mathbb{Z}}^{p-1,-1}(Y, \mathcal{O} \cap Y)$. Clearly, a relative integer Čech cochain is also a relative real Čech cochain. A $Y$ relative integer Čech $p$-cochain $\left(\hat{\Xi}^{p,-1}, \hat{\Upsilon}^{p-1,-1}\right)$ of $X$ is a cocycle if it satisfies eq. (1.2.15) with $\left(\Xi^{p,-1}, \Upsilon^{p-1,-1}\right)$ replaced by $\left(\hat{\Xi}^{p,-1}, \hat{\Upsilon}^{p-1,-1}\right)$, so that it is a cocycle also when seen as a relative real Čech cochain. A $Y$ relative integer Čech $p$-cocycle $\left(\hat{\Xi}^{p,-1}, \hat{\Upsilon}^{p-1,-1}\right)$ of $X$ is a coboundary if it satisfies eq. (1.2.16) with $\left(\xi^{p-1,-1}, v^{p-2,-1}\right)$ replaced by any $Y$ relative integer Čech $p-1-$ cochain $\left(\hat{\xi}^{p-1,-1}, \hat{v}^{p-2,-1}\right)$, so that it is a coboundary also when seen as a relative real Cech cochain. 
We denote by $C_{C \mathbb{Z}}^{p}(X, Y, \mathcal{O}), Z_{C \mathbb{Z}}^{p}(X, Y, \mathcal{O}), B_{C \mathbb{Z}}^{p}(X, Y, \mathcal{O})$ the groups of $Y$ relative integer Čech $p$-cochains, cocycles and coboundaries of $X$, respectively. Two relative integer $p$ cocycles are equivalent if their difference is a integer $p$-coboundary. The equivalence classes of $Y$ relative integer Čech $p$-cocycles of $X$ form the $p$-th relative integer Čech cohomology group $H_{C \mathbb{Z}}^{p}(X, Y, \mathcal{O})$.

A $Y$ relative differential $p$-cocycle of $X$ is a Čech six-tuple $\left(\Xi^{p,-1}, \Upsilon^{p-1,-1} ; \Xi^{* p-1,-1}\right.$, $\left.\Upsilon^{* p-2,-1} ; \hat{\Xi}^{p,-1}, \hat{\Upsilon}^{p-1,-1}\right)$, where $\Xi^{p,-1} \in \mathcal{C}^{p,-1}(X, \mathcal{O}), \Upsilon^{p-1,-1} \in \mathcal{C}^{p-1,-1}(Y, \mathcal{O} \cap Y)$, $\Xi^{* p-1,-1} \in \mathcal{C}^{p-1,-1}(X, \mathcal{O}), \Upsilon^{* p-2,-1} \in \mathcal{C}^{p-2,-1}(Y, \mathcal{O} \cap Y), \hat{\Xi}^{p,-1} \in \mathcal{C}_{\mathbb{Z}}^{p,-1}(X, \mathcal{O}), \hat{\Upsilon}^{p-1,-1}$ $\in \mathcal{C}_{\mathbb{Z}}^{p-1,-1}(Y, \mathcal{O} \cap Y)$, satisfying

$$
\begin{aligned}
& \delta \Xi^{p,-1}=0, \\
& i^{*} \Xi^{p,-1}-\delta \Upsilon^{p-1,-1}=0, \\
& \delta \Xi^{* p-1,-1}=\hat{\Xi}^{p,-1}-\Xi^{p,-1}, \\
& i^{*} \Xi^{* p-1,-1}-\delta \Upsilon^{* p-2,-1}=\hat{\Upsilon}^{p-1,-1}-\Upsilon^{p-1,-1}, \\
& \delta \hat{\Xi}^{p,-1}=0, \\
& i^{*} \hat{\Xi}^{p,-1}-\delta \hat{\Upsilon}^{p-1,-1}=0 .
\end{aligned}
$$

Note that $\left(\Xi^{p,-1}, \Upsilon^{p-1,-1}\right) \in Z_{C}^{p}(X, Y, \mathcal{O})$ and $\left(\hat{\Xi}^{p,-1}, \hat{\Upsilon}^{p-1,-1}\right) \in Z_{C \mathbb{Z}}^{p}(X, Y, \mathcal{O})$ (cfr. eq. (1.2.15)). A $Y$ relative differential $p$-cocycle $\left(\Xi^{p,-1}, \Upsilon^{p-1,-1} ; \Xi^{* p-1,-1}, \Upsilon^{* p-2,-1} ; \hat{\Xi}^{p,-1}\right.$, $\hat{\Upsilon}^{p-1,-1}$ ) of $X$ is a differential coboundary if

$$
\begin{aligned}
& \Xi^{p,-1}=\delta \xi^{p-1,-1} \\
& \Upsilon^{p-1,-1}=i^{*} \xi^{p-1,-1}-\delta v^{p-2,-1}, \\
& \Xi^{* p-1,-1}=\hat{\xi}^{p-1,-1}-\xi^{p-1,-1}, \\
& \Upsilon^{* p-2,-1}=\hat{v}^{p-2,-1}-v^{p-2,-1}, \\
& \hat{\Xi}^{p-1}=\delta \hat{\xi}^{p-1,-1} \\
& \hat{\Upsilon}^{p-1,-1}=i^{*} \hat{\xi}^{p-1,-1}-\delta \hat{v}^{p-2,-1},
\end{aligned}
$$


where $\xi^{p-1,-1} \in \mathcal{C}^{p-1,-1}(X, \mathcal{O}), v^{p-2,-1} \in \mathcal{C}^{p-2,-1}(Y, \mathcal{O} \cap Y), \hat{\xi}^{p-1,-1} \in \mathcal{C}_{\mathbb{Z}}^{p-1,-1}(X, \mathcal{O})$, $\hat{v}^{p-2,-1} \in \mathcal{C}_{\mathbb{Z}}^{p-2,-1}(Y, \mathcal{O} \cap Y)$. We denote by $Z D_{C}^{p}(X, Y, \mathcal{O}), B D_{C}^{p}(X, Y, \mathcal{O})$ the groups of $Y$ relative differential $p$-cocycles and coboundaries of $X$, respectively. Two relative differential $p$-cocycles are equivalent if their difference is a differential coboundary. The equivalence classes of $Y$ relative differential $p$-cocycle of $X$ form a group $H D_{C}^{p}(X, Y, \mathcal{O})$. An analogous notion of differential cocycle has been introduced for the absolute case in $[15]$.

A $Y$ relative differential $p$-cocycle $\left(\Xi^{p,-1}, \Upsilon^{p-1,-1} ; \Xi^{* p-1,-1}, \Upsilon^{* p-2,-1} ; \hat{\Xi}^{p,-1}, \hat{\Upsilon}^{p-1,-1}\right)$ of $X$ is torsion if it is of the form (1.3.4)-(1.3.6) with $\hat{\xi}^{p-1,-1} \in \mathcal{C}^{p-1,-1}(X, \mathcal{O}), \hat{v}^{p-2,-1} \in$ $\mathcal{C}^{p-2,-1}(Y, \mathcal{O} \cap Y)$ subject to the condition $\delta \hat{\xi}^{p-1,-1} \in \mathcal{C}_{\mathbb{Z}}^{p,-1}(X, \mathcal{O}), i^{*} \hat{\xi}^{p-1,-1}-\delta \hat{v}^{p-2,-1} \in$ $\mathcal{C}_{\mathbb{Z}}^{p-1,-1}(Y, \mathcal{O} \cap Y)$. Torsion differential cocycles form a subgroup $Z D_{C t}^{p}(X, Y, \mathcal{O})$ of $Z D_{C}^{p}(X$, $Y, \mathcal{O})$. Being invariant under translation by $B D_{C}^{p}(X, Y, \mathcal{O}), Z D_{C t}^{p}(X, Y, \mathcal{O})$ projects to a subgroup $H D_{C t}^{p}(X, Y, \mathcal{O})$ of $H D_{C}^{p}(X, Y, \mathcal{O})$.

\section{Relative homology and $\mathcal{O}$-small homology isomorphism and the relative Čech singular/} Čech-de Rham isomorphisms

The barycentric subdivision operator $q$ (cfr. subsect. 1.1) acts on relative chains in obvious fashion. For any relative chain $\left(S_{p-1}, T_{p-2}\right) \in C_{p-1}^{s}(X, Y)$, there is an integer $k\left(S_{p-1}, T_{p-2}, \mathcal{O}\right) \geq 0$ such that $\left(q^{k} S_{p-1}, q^{k} T_{p-2}\right) \in C_{p-1}^{s \mathcal{O}}(X, Y)$ is $\mathcal{O}$-small for $k \geq k\left(S_{p-1}, T_{p-2}, \mathcal{O}\right)$. By the chain relation $(1.1 .5)$, if $\left(S_{p-1}, T_{p-2}\right) \in Z_{p-1}^{s}(X, Y)$ is a relative cycle, then $\left(q^{k} S_{p-1}, q^{k} T_{p-2}\right) \in Z_{p-1}^{s \mathcal{O}}(X, Y)$ also is. If $\left(S_{p-1}, T_{p-2}\right) \in B_{p-1}^{s}(X, Y)$ is a relative boundary, then $\left(q^{k} S_{p-1}, q^{k} T_{p-2}\right) \in B_{p-1}^{s \mathcal{O}}(X, Y)$ also is and the corresponding relative chain $\left(q^{k} s_{p}, q^{k} t_{p-1}\right)$ is $\mathcal{O}$-small for $k$ large enough (cfr. eq. (1.2.2)). Using the chain relation (1.1.5) and the homotopy relation (1.1.6), it is possible to construct a chain equivalence of the complex of $Y$ relative singular chains and that of $\mathcal{O}$-small $Y$ relative singular chains for any open covering $\mathcal{O}$ of $X$ [36]. Hence, the corresponding homologies are isomorphic

$$
H_{p-1}^{s}(X, Y) \cong H_{p-1}^{s \mathcal{O}}(X, Y)
$$

We say that the open covering $\mathcal{O}$ of $X$ is a good covering of the pair $X, Y$, if $\mathcal{O}$ is a good covering of $X$ and $\mathcal{O} \cap Y$ is good a covering of $Y$. (See appendix A1.)

An $\mathcal{O}$-small $Y$ relative singular $p-1$-cycle $\left(S_{p-1}, T_{p-2}\right) \in Z_{p-1}^{s \mathcal{O}}(X, Y)$ and a $Y$ relative integer Čech $p$-cycle $\left(S_{p-1,-1}, T_{p-2,-1}\right) \in Z_{p-1}^{C}(X, Y, \mathcal{O})$ are said compatible if they fit into 
some $Y$ relative Čech singular $p$-intertwiner $\left(S_{-1, p-1}, T_{-1, p-2} ;\left\{V_{k, p-1-k}\right\},\left\{Z_{k, p-2-k}\right\}\right.$; $\left.S_{p-1,-1}, T_{p-2,-1}\right) \in Z I_{p-1}^{C s}(X, Y, \mathcal{O})$ (cfr. eqs. (1.2.1), (1.2.3), (1.2.5)-(1.2.7)). From eqs. (1.2.2), (1.2.4), (1.2.8)-(1.2.10), it follows that any $\mathcal{O}$-small $Y$ relative singular $p$ - 1-boundary $\left(S_{p-1}, T_{p-2}\right) \in B_{p-1}^{s \mathcal{O}}(X, Y)$ is always compatible with any $Y$ relative integer Čech $p$-boundary $\left(S_{p-1,-1}, T_{p-2,-1}\right) \in B_{p-1}^{C}(X, Y, \mathcal{O})$ through a trivial intertwiner in $B I_{p-1}^{C s}(X, Y, \mathcal{O})$. Therefore, the compatibility relation in $Z_{p-1}^{s \mathcal{O}}(X, Y) \times Z_{p-1}^{C}(X, Y, \mathcal{O})$ defined above induces a compatibility relation in $H_{p-1}^{s \mathcal{O}}(X, Y) \times H_{p-1}^{C}(X, Y, \mathcal{O})$ at the level of relative homology. A fundamental theorem states that, when $\mathcal{O}$ is a good covering of the pair $X, Y$, this relation is actually an isomorphism

$$
H_{p-1}^{s \mathcal{O}}(X, Y) \cong H_{p-1}^{C}(X, Y, \mathcal{O})
$$

Its proof is analogous to that of the absolute case [34]. On account of the isomorphism (1.4.1), we find out that, for such coverings, $H_{p-1}^{C}(X, Y, \mathcal{O})$ does not depend on $\mathcal{O}$ up to isomorphism.

A $Y$ relative de Rham $p$-cocycle $\left(\Xi^{p}, \Upsilon^{p-1}\right) \in Z_{d R}^{p}(X, Y)$ and a $Y$ relative real Čech $p$ cocycle $\left(\Xi^{p,-1}, \Upsilon^{p-1,-1}\right) \in Z_{C}^{p}(X, Y, \mathcal{O})$ are said compatible if they fit into some $Y$ relative Čech-de Rham $p$-cointertwiner $\left(\Xi^{-1, p}, \Upsilon^{-1, p-1} ;\left\{\Omega^{k, p-1-k}\right\},\left\{\Theta^{k, p-2-k}\right\} ; \Xi^{p,-1}, \Upsilon^{p-1,-1}\right)$ $\in Z I_{C d R}^{p}(X, Y, \mathcal{O})$ (cfr. eqs. (1.2.13), (1.2.15), (1.2.17)-(1.2.19)). From eqs. (1.2.14), (1.2.16), (1.2.20)-(1.2.22), it follows that a $Y$ relative de Rham $p$-coboundary $\left(\Xi^{p}, \Upsilon^{p-1}\right) \in$ $B_{d R}^{p}(X, Y)$ is compatible with any $Y$ relative real Čech $p$-coboundary $\left(\Xi^{p,-1}, \Upsilon^{p-1,-1}\right)$ $\in B_{C}^{p}(X, Y, \mathcal{O})$ through a trivial cointertwiner in $B I_{C d R}^{p}(X, Y, \mathcal{O})$. Therefore, the compatibility relation in $Z_{d R}^{p}(X, Y) \times Z_{C}^{p}(X, Y, \mathcal{O})$ defined above induces a compatibility relation in $H_{d R}^{p}(X, Y) \times H_{C}^{p}(X, Y, \mathcal{O})$ at the level of relative cohomology. A fundamental theorem states that, when $\mathcal{O}$ is a good covering of the pair $X, Y$, this relation is actually an isomorphism

$$
H_{d R}^{p}(X, Y) \cong H_{C}^{p}(X, Y, \mathcal{O}),
$$

so that for such coverings $H_{C}^{p}(X, Y, \mathcal{O})$ does not depend on $\mathcal{O}$ up to isomorphism. Again, the proof is analogous to that of the absolute case [34].

\section{Integrality in relative cohomology}

As is well-known, given any Abelian group $G$, by dualization via the functor $\operatorname{Hom}_{\mathbb{Z}}(\cdot$, $G$ ) of the singular chain complex of a manifold $M$, one can construct the singular cochain 
complex of $M$ with coefficients in $G$. When an open covering $\mathcal{O}$ of $M$ is given, one can similarly define $\mathcal{O}_{-}$-small singular cochains and Čech singular cochains with coefficients in $G$. This allows one to set up a cohomological framework that parallels completely the original homological one. (See refs. [34,36] for background material.) The generalization to the relative case is straightforward.

Proceeding as outlined above, it is possible to introduce the relative real singular cohomology space $H_{s}^{p}(X, Y)$ and the relative integer singular cohomology group $H_{s \mathbb{Z}}^{p}(X, Y)$.

The natural inclusion of the group of relative integer singular cochains into the space of relative real singular cochains is a cochain map. Thus, there is a canonical homomorphism $H_{s \mathbb{Z}}^{p}(X, Y) \rightarrow H_{s}^{p}(X, Y)$ of the relative singular cohomology. Its kernel $\operatorname{Tor}_{s}^{p}(X, Y)$ is the relative singular torsion subgroup of $H_{s \mathbb{Z}}^{p}(X, Y)$. Its range $\tilde{H}_{s \mathbb{Z}}^{p}(X, Y)$ is the relative integer singular cohomology lattice of $H_{s}^{p}(X, Y)$.

The above setting has faithful translation in relative Čech cohomology. Let $\mathcal{O}$ be a covering of $X$. The inclusion $C_{C \mathbb{Z}}^{p}(X, Y, \mathcal{O}) \rightarrow C_{C}^{p}(X, Y, \mathcal{O})$ is a cochain map (cfr. subsect. 1.3). Thus, it induces a homomorphism $H_{C \mathbb{Z}}^{p}(X, Y, \mathcal{O}) \rightarrow H_{C}^{p}(X, Y, \mathcal{O})$ of the relative integer Čech cohomology group into the relative real Čech cohomology space. Its kernel $\operatorname{Tor}_{C}^{p}(X, Y, \mathcal{O})$ is the relative Cech torsion subgroup of $H_{C \mathbb{Z}}^{p}(X, Y, \mathcal{O})$. Its range $\tilde{H}_{C \mathbb{Z}}^{p}(X, Y, \mathcal{O})$ is the relative integer Cech cohomology lattice of $H_{C}^{p}(X, Y, \mathcal{O})$.

If $\mathcal{O}$ is restricted to be a good open covering of $X, Y$ (cfr. subsect. 1.4), then relative singular cohomology and relative Čech cohomology are completely isomorphic:

$$
\begin{aligned}
H_{s \mathbb{Z}}^{p}(X, Y) & \cong H_{C \mathbb{Z}}^{p}(X, Y, \mathcal{O}), \\
\operatorname{Tor}_{s}^{p}(X, Y) & \cong \operatorname{Tor}_{C}^{p}(X, Y, \mathcal{O}), \\
H_{s}^{p}(X, Y) & \cong H_{C}^{p}(X, Y, \mathcal{O}), \\
\tilde{H}_{s \mathbb{Z}}^{p}(X, Y) & \cong \tilde{H}_{C \mathbb{Z}}^{p}(X, Y, \mathcal{O}) .
\end{aligned}
$$

The above isomorphisms are consistent: the isomorphisms (1.5.2), (1.5.4) are the restriction the isomorphisms (1.5.1), (1.5.3), respectively. Further, the homomorphism $H_{C \mathbb{Z}}^{p}(X, Y, \mathcal{O})$ $\rightarrow H_{C}^{p}(X, Y, \mathcal{O})$ is obtained by the composition of the homomorphism $H_{s \mathbb{Z}}^{p}(X, Y) \rightarrow$ $H_{s}^{p}(X, Y)$ with the isomorphisms $(1.5 .1),(1.5 .3)$. The proofs are formally analogous to that of the isomorphism (1.4.3), though extra work must be done to show the isomorphism of $\mathcal{O}$-small relative singular cohomology and relative singular cohomology. Note that, by (1.5.1)-(1.5.4), $H_{C \mathbb{Z}}^{p}(X, Y, \mathcal{O}), \operatorname{Tor}_{C}^{p}(X, Y, \mathcal{O}), H_{C}^{p}(X, Y, \mathcal{O}), \tilde{H}_{C \mathbb{Z}}^{p}(X, Y, \mathcal{O})$ are all independent from the good open covering $\mathcal{O}$ up to isomorphism. 
Let $\mathcal{O}$ be a good open covering of $X, Y$. A $Y$ relative real Čech $p$-cocycle $\left(\Xi^{p,-1}\right.$, $\left.\Upsilon^{p-1,-1}\right) \in Z_{C}^{p}(X, Y, \mathcal{O})$ is said cohomologically integer if it fits into some $Y$ relative differential $p$-cocycle $\left(\Xi^{p,-1}, \Upsilon^{p-1,-1} ; \Xi^{* p-1,-1}, \Upsilon^{* p-2,-1} ; \hat{\Xi}^{p,-1}, \hat{\Upsilon}^{p-1,-1}\right) \in Z D_{C}^{p}(X, Y, \mathcal{O})$ (cfr. eqs. (1.2.15), (1.3.1)-(1.3.3)). From eqs. (1.2.16), (1.3.4)-(1.3.6), it follows that any $Y$ relative real Čech $p$-coboundary $\left(\Xi^{p,-1}, \Upsilon^{p-1,-1}\right) \in B_{C}^{p}(X, Y, \mathcal{O})$ is always cohomologically integer, being part of a differential coboundary in $B D_{C}^{p}(X, Y, \mathcal{O})$. We denote by $\tilde{Z}_{C Z}^{p}(X, Y, \mathcal{O})$ the subgroup of $Z_{C}^{p}(X, Y, \mathcal{O})$ formed by the cohomologically integer relative real Čech $p$-cocycles. Being invariant under translation by $B_{C}^{p}(X, Y, \mathcal{O}), \tilde{Z}_{C \mathbb{Z}}^{p}(X, Y, \mathcal{O})$ projects to a lattice of $H_{C}^{p}(X, Y, \mathcal{O})$. Clearly, $Z_{C \mathbb{Z}}^{p}(X, Y, \mathcal{O}) \subseteq \tilde{Z}_{C \mathbb{Z}}^{p}(X, Y, \mathcal{O})$ (cfr. subsect. 1.3) and the lattice mentioned is precisely the relative integer Cech cohomology lattice $\tilde{H}_{C \mathbb{Z}}^{p}(X, Y, \mathcal{O})$ introduced above.

A $Y$ relative de Rham $p$-cocycle $\left(\Xi^{p}, \Upsilon^{p-1}\right) \in Z_{d R}^{p}(X, Y)$ is said cohomologically integer, if it is compatible with some cohomologically integer $Y$ relative real Čech $p$ cocycle $\left(\Xi^{p,-1}, \Upsilon^{p-1,-1}\right) \in \tilde{Z}_{C \mathbb{Z}}^{p}(X, Y, \mathcal{O})$ for some good open covering $\mathcal{O}$ of $X, Y$ (cfr. subsect. 1.4). A $Y$ relative de Rham $p$-coboundary $\left(\Xi^{p}, \Upsilon^{p-1}\right) \in B_{d R}^{p}(X, Y)$ is always cohomologically integer, since it is compatible with a $Y$ relative real Čech $p$-coboundary $\left(\Xi^{p,-1}, \Upsilon^{p-1,-1}\right) \in B_{C}^{p}(X, Y, \mathcal{O})$, which is necessarily cohomologically integer, for any good open covering $\mathcal{O}$. We denote by $Z_{d R \mathbb{Z}}^{p}(X, Y)$ be the subgroup of $Z_{d R}^{p}(X, Y)$ formed by the cohomologically integer relative de Rham $p$-cocycles. Since $Z_{d R \mathbb{Z}}^{p}(X, Y)$ is invariant under translation by $B_{d R}^{p}(X, Y), Z_{d R \mathbb{Z}}^{p}(X, Y)$ projects to a lattice subgroup $H_{d R \mathbb{Z}}^{p}(X, Y)$ of $H_{d R}^{p}(X, Y)$. For any fixed good covering $\mathcal{O}$, every cohomologically integer de Rham $p$ cocycle $\left(\Xi^{p}, \Upsilon^{p-1}\right) \in Z_{d R \mathbb{Z}}^{p}(X, Y)$ is compatible with a cohomologically integer $Y$ relative real Čech $p$-cocycle $\left(\Xi^{p,-1}, \Upsilon^{p-1,-1}\right) \in \tilde{Z}_{C Z \mathbb{Z}}^{p}(X, Y, \mathcal{O})$. Further, $H_{d R \mathbb{Z}}^{p}(X, Y)$ corresponds precisely to the relative integer Čech cohomology lattice $\tilde{H}_{C \mathbb{Z}}^{p}(X, Y, \mathcal{O})$ under the Čech-de Rham isomorphism (1.4.3).

From (1.4.3), (1.5.3), (1.5.4) and the above discussion, one deduces the isomorphisms

$$
\begin{gathered}
H_{d R}^{p}(X, Y) \cong H_{s}^{p}(X, Y), \\
H_{d R \mathbb{Z}}^{p}(X, Y) \cong \tilde{H}_{s \mathbb{Z}}^{p}(X, Y),
\end{gathered}
$$

the isomorphism (1.5.6) being the restriction of that (1.5.5). 


\section{The relative Cheeger-Simons differential characters}

Let $p, X, Y$ satisfy the assumptions stated at the end of subsect. 1.1 and let $\mathcal{O}$ be a good covering of $X, Y$ (cfr. subsect. 1.4).

\section{Construction of the maps $I_{1}^{\mathcal{O}}$ and $I_{2}^{\mathcal{O}}$}

We now define two basic realvalued functions, $I_{1}^{\mathcal{O}}, I_{2}^{\mathcal{O}}$, of the appropriate relative data. In view of the construction of relative Cheeger-Simons differential characters, we analyze in detail the properties of $I_{1}^{\mathcal{O}}, I_{2}^{\mathcal{O}}$, when the relative data are varied by trivial amounts. Here, we systematically use the notation (1.1.15) for conciseness.

The first function, $I_{1}^{\mathcal{O}}$, depends on the following relative data: a relative Čech singular $p-1$-intertwiner $\left(S_{-1, p-1}, T_{-1, p-2} ;\left\{V_{k, p-1-k} \mid k \in I_{p-1}\right\},\left\{Z_{k, p-2-k} \mid k \in I_{p-2}\right\} ; S_{p-1,-1}\right.$, $\left.T_{p-2,-1}\right) \in Z I_{p-1}^{C s}(X, Y, \mathcal{O})$ (cfr. eqs. (1.2.5)-(1.2.7)); a relative Čech-de Rham $p$-cointertwiner $\left(\Xi^{-1, p}, \Upsilon^{-1, p-1} ;\left\{\Omega^{k, p-1-k} \mid k \in I^{p-1}\right\},\left\{\Theta^{k, p-2-k} \mid k \in I^{p-2}\right\} ; \Xi^{p,-1}, \Upsilon^{p-1,-1}\right) \in$ $Z I_{C d R}^{p}(X, Y, \mathcal{O})$ (cfr. eqs. (1.2.17)-(1.2.19)). It is given by

$$
I_{1}^{\mathcal{O}}=\sum_{k=0}^{p-1}(-1)^{k}\left\langle V_{k, p-1-k}, \Omega^{k, p-1-k}\right\rangle-\sum_{k=0}^{p-2}(-1)^{k}\left\langle Z_{k, p-2-k}, \Theta^{k, p-2-k}\right\rangle .
$$

When the relative arguments are varied by arbitrary amounts (generically denoted by $\Delta)$ the variation $\Delta I_{1}^{\mathcal{O}}$ of $I_{1}^{\mathcal{O}}$ is given by

$$
\begin{aligned}
\Delta I_{1}^{\mathcal{O}}= & \sum_{k=0}^{p-1}(-1)^{k}\left\langle V_{k, p-1-k}, \Delta \Omega^{k, p-1-k}\right\rangle-\sum_{k=0}^{p-2}(-1)^{k}\left\langle Z_{k, p-2-k}, \Delta \Theta^{k, p-2-k}\right\rangle \\
& +\sum_{k=0}^{p-1}(-1)^{k}\left\langle\Delta V_{k, p-1-k}, \Omega^{k, p-1-k}\right\rangle-\sum_{k=0}^{p-2}(-1)^{k}\left\langle\Delta Z_{k, p-2-k}, \Theta^{k, p-2-k}\right\rangle \\
& +\sum_{k=0}^{p-1}(-1)^{k}\left\langle\Delta V_{k, p-1-k}, \Delta \Omega^{k, p-1-k}\right\rangle-\sum_{k=0}^{p-2}(-1)^{k}\left\langle\Delta Z_{k, p-2-k}, \Delta \Theta^{k, p-2-k}\right\rangle .
\end{aligned}
$$

If $\left(\Delta S_{-1, p-1}, \Delta T_{-1, p-2} ;\left\{\Delta V_{k, p-1-k}\right\},\left\{\Delta Z_{k, p-2-k}\right\} ; \Delta S_{p-1,-1}, \Delta T_{p-2,-1}\right) \in B I_{p-1}^{C s}(X, Y$, $\mathcal{O})$ is a trivial relative intertwiner (cfr. eqs $(1.2 .8)-(1.2 .10))$ and $\left(\Delta \Xi^{-1, p}, \Delta \Upsilon^{-1, p-1}\right.$; $\left.\left\{\Delta \Omega^{k, p-1-k}\right\},\left\{\Delta \Theta^{k, p-2-k}\right\} ; \Delta \Xi^{p,-1}, \Delta \Upsilon^{p-1,-1}\right) \in B I_{C d R}^{p}(X, Y, \mathcal{O})$ is a trivial relative cointertwiner (cfr. eqs. (1.2.20)-(1.2.22)), one has

$$
\Delta I_{1}^{\mathcal{O}}=\left\langle S_{-1, p-1}, \xi^{-1, p-1}\right\rangle-\left\langle T_{-1, p-2}, v^{-1, p-2}\right\rangle
$$




$$
\begin{aligned}
& +\left\langle s_{-1, p}, \Xi^{-1, p}\right\rangle-\left\langle t_{-1, p-1}, \Upsilon^{-1, p-1}\right\rangle \\
& +\left\langle s_{-1, p}, d \xi^{-1, p-1}\right\rangle-\left\langle t_{-1, p-1}, i^{*} \xi^{-1, p-1}-d v^{-1, p-2}\right\rangle \\
& +(-1)^{p-1}\left[\left\langle S_{p-1,-1}, \xi^{p-1,-1}\right\rangle-\left\langle T_{p-2,-1}, v^{p-2,-1}\right\rangle\right. \\
& +\left\langle s_{p,-1}, \Xi^{p,-1}\right\rangle-\left\langle t_{p-1,-1}, \Upsilon^{p-1,-1}\right\rangle \\
& \left.+\left\langle s_{p,-1}, \delta \xi^{p-1,-1}\right\rangle-\left\langle t_{p-1,-1}, i^{*} \xi^{p-1,-1}-\delta v^{p-2,-1}\right\rangle\right]
\end{aligned}
$$

The second function, $I_{2}^{\mathcal{O}}$, depends on the following relative data: a relative integer Čech $p-1$-cycle $\left(S_{p-1,-1}, T_{p-2,-1}\right) \in Z_{p-1}^{C}(X, Y, \mathcal{O})$ (cfr. eq. (1.2.3)); a relative differential $p$-cocycle $\left(\Xi^{p,-1}, \Upsilon^{p-1,-1} ; \Xi^{* p-1,-1}, \Upsilon^{* p-2,-1} ; \hat{\Xi}^{p,-1}, \hat{\Upsilon}^{p-1,-1}\right) \in Z D_{C}^{p}(X, Y, \mathcal{O})$ (cfr. eqs. (1.3.1)-(1.3.3)). It is given by

$$
I_{2}^{\mathcal{O}}=(-1)^{p-1}\left[\left\langle S_{p-1,-1}, \Xi^{* p-1,-1}\right\rangle-\left\langle T_{p-2,-1}, \Upsilon^{* p-2,-1}\right\rangle\right]
$$

When the relative arguments are varied by arbitrary amounts (again generically denoted by $\Delta$ ), the variation $\Delta I_{2}^{\mathcal{O}}$ of $I_{2}^{\mathcal{O}}$ is given by

$$
\begin{aligned}
\Delta I_{2}^{\mathcal{O}}= & (-1)^{p-1}\left[\left\langle S_{p-1,-1}, \Delta \Xi^{* p-1,-1}\right\rangle-\left\langle T_{p-2,-1}, \Delta \Upsilon^{* p-2,-1}\right\rangle\right. \\
& +\left\langle\Delta S_{p-1,-1}, \Xi^{* p-1,-1}\right\rangle-\left\langle\Delta T_{p-2,-1}, \Upsilon^{* p-2,-1}\right\rangle \\
& \left.+\left\langle\Delta S_{p-1,-1}, \Delta \Xi^{* p-1,-1}\right\rangle-\left\langle\Delta T_{p-2,-1}, \Delta \Upsilon^{* p-2,-1}\right\rangle\right]
\end{aligned}
$$

If $\left(\Delta S_{p-1,-1}, \Delta T_{p-2,-1}\right) \in B_{p-1}^{C}(X, Y, \mathcal{O})$ is a relative Čech boundary (cfr. eq. (1.2.2)) and $\left(\Delta \Xi^{p,-1}, \Delta \Upsilon^{p-1,-1} ; \Delta \Xi^{* p-1,-1}, \Delta \Upsilon^{* p-2,-1} ; \Delta \hat{\Xi}^{p,-1}, \Delta \hat{\Upsilon}^{p-1,-1}\right) \in B D_{C}^{p}(X, Y, \mathcal{O})$ or $Z D_{C t}^{p}(X, Y, \mathcal{O})$ is either a relative differential coboundary or a torsion relative differential cocycle (cfr. eqs. (1.3.4)-(1.3.6)), then

$$
\begin{aligned}
\Delta I_{2}^{\mathcal{O}}= & -(-1)^{p-1}\left[\left\langle S_{p-1,-1}, \xi^{p-1,-1}\right\rangle-\left\langle T_{p-2,-1}, v^{p-2,-1}\right\rangle\right. \\
& +\left\langle s_{p,-1}, \Xi^{p,-1}\right\rangle-\left\langle t_{p-1,-1}, \Upsilon^{p-1,-1}\right\rangle \\
& \left.+\left\langle s_{p,-1}, \delta \xi^{p-1,-1}\right\rangle-\left\langle t_{p-1,-1}, i^{*} \xi^{p-1,-1}-\delta v^{p-2,-1}\right\rangle\right]
\end{aligned}
$$




$$
\begin{aligned}
& +(-1)^{p-1}\left[\left\langle S_{p-1,-1}, \hat{\xi}^{p-1,-1}\right\rangle-\left\langle T_{p-2,-1}, \hat{v}^{p-2,-1}\right\rangle\right. \\
& +\left\langle s_{p,-1}, \hat{\Xi}^{p,-1}\right\rangle-\left\langle t_{p-1,-1}, \hat{\Upsilon}^{p-1,-1}\right\rangle \\
& \left.+\left\langle s_{p,-1}, \delta \hat{\xi}^{p-1,-1}\right\rangle-\left\langle t_{p-1,-1}, i^{*} \hat{\xi}^{p-1,-1}-\delta \hat{v}^{p-2,-1}\right\rangle\right] .
\end{aligned}
$$

Let $\left(S_{p-1}, T_{p-2}\right) \in Z_{p-1}^{s \mathcal{O}}(X, Y),\left(\Xi^{p}, \Upsilon^{p-1}\right) \in Z_{d R \mathbb{Z}}^{p}(X, Y)$ be respectively an $\mathcal{O}$-small relative singular $p-1$-cycle (cfr. eq. (1.2.1) and subsect. 1.2) and a cohomologically integer relative de Rham $p$-cocycle, (cfr. eq. (1.2.13) and subsect. 1.5). From the discussion of subsects. 1.4 and 1.5, we can carry out the following construction.

$\left(S_{p-1}, T_{p-2}\right)$ can be extended to some relative Čech singular $p-1$-intertwiner $\left(S_{-1, p-1}\right.$, $\left.T_{-1, p-2} ;\left\{V_{k, p-1-k}\right\},\left\{Z_{k, p-2-k}\right\} ; S_{p-1,-1}, T_{p-2,-1}\right) \in Z I_{p-1}^{C s}(X, Y, \mathcal{O})$ (cfr. eqs. (1.2.5)(1.2.7) and subsect. 1.4). By standard Čech singular techniques, one easily sees that the intertwiner $\left(S_{-1, p-1}, T_{-1, p-2} ;\left\{V_{k, p-1-k}\right\},\left\{Z_{k, p-2-k}\right\} ; S_{p-1,-1}, T_{p-2,-1}\right)$ is defined up to a trivial relative Čech singular intertwiner of the form $(1.2 .8)-(1.2 .10)$ with $s_{-1, p}=0$, $t_{-1, p-1}=0$.

$\left(\Xi^{p}, \Upsilon^{p-1}\right)$ can be extended to some relative Čech-de Rham $p$-cointertwiner $\left(\Xi^{-1, p}\right.$, $\left.\Upsilon^{-1, p-1} ;\left\{\Omega^{k, p-1-k}\right\},\left\{\Theta^{k, p-2-k}\right\} ; \Xi^{p,-1}, \Upsilon^{p-1,-1}\right) \in Z I_{C d R}^{p}(X, Y, \mathcal{O})$ (cfr. eqs. (1.2.17)(1.2.19) and subsect. 1.4). By the standard Čech-de Rham techniques, it is easy to see that the cointertwiner $\left(\Xi^{-1, p}, \Upsilon^{-1, p-1} ;\left\{\Omega^{k, p-1-k}\right\},\left\{\Theta^{k, p-2-k}\right\} ; \Xi^{p,-1}, \Upsilon^{p-1,-1}\right)$ is defined up to a trivial relative Cech-de Rham cointertwiner of the form (1.2.20)-(1.2.22) with $\xi^{-1, p-1}=0, v^{-1, p-2}=0$.

As $\left(\Xi^{p}, \Upsilon^{p-1}\right)$ is cohomologically integer, the relative real Čech $p$-cocycle $\left(\Xi^{p,-1}\right.$, $\left.\Upsilon^{p-1,-1}\right) \in \tilde{Z}_{C \mathbb{Z}}^{p}(X, Y, \mathcal{O})$ is cohomologically integer as well (cfr. subsects. 1.5). Then, $\left(\Xi^{p,-1}, \Upsilon^{p-1,-1}\right)$ fits into some relative differential $p$-cocycle $\left(\Xi^{p,-1}, \Upsilon^{p-1,-1} ; \Xi^{* p-1,-1}\right.$, $\left.\Upsilon^{* p-2,-1} ; \hat{\Xi}^{p,-1}, \hat{\Upsilon}^{p-1,-1}\right) \in Z D_{C}^{p}(X, Y, \mathcal{O})$ (cfr. eqs. (1.3.1)-(1.3.3) and subsect. 1.5). As $\left(\Xi^{p,-1}, \Upsilon^{p-1,-1}\right)$ is defined only up to a relative real Čech coboundary of the form $(1.2 .16)$, the relative differential cocycle $\left(\Xi^{p,-1}, \Upsilon^{p-1,-1} ; \Xi^{* p-1,-1}, \Upsilon^{* p-2,-1} ; \hat{\Xi}^{p,-1}, \hat{\Upsilon}^{p-1,-1}\right)$ is determined up to a torsion relative differential cocycle of the form (1.3.4)-(1.3.6). Indeed, when the relative Čech torsion $\operatorname{Tor}_{C}^{p}(X, Y, \mathcal{O})$ is non vanishing, the cohomology class of the relative integer Čech cocycle $\left(\hat{\Xi}^{p,-1}, \hat{\Upsilon}^{p-1,-1}\right)$ in $H_{C \mathbb{Z}}^{p}(X, Y, \mathcal{O})$ is not uniquely fixed by that of the relative real Čech cocycle $\left(\Xi^{p,-1}, \Upsilon^{p-1,-1}\right)$ in $H_{C}^{p}(X, Y, \mathcal{O})$ and, thus, the ambiguity of the relative differential cocycle is not in general a relative differential coboundary. 
Using the relative homological and cohomological data obtained in this way from $\left(S_{p-1}, T_{p-2}\right)$ and $\left(\Xi^{p}, \Upsilon^{p-1}\right)$, we set

$$
I^{\mathcal{O}}=I_{1}^{\mathcal{O}}+I_{2}^{\mathcal{O}}
$$

Since, however. those data are not determined by $\left(S_{p-1}, T_{p-2}\right)$ and $\left(\Xi^{p}, \Upsilon^{p-1}\right)$ in unique fashion, as explained above, $I^{\mathcal{O}}$ is affected by an ambiguity $\Delta I^{\mathcal{O}}$ which we are now going to compute.

From the above discussion, by inspection of (2.1.3), (2.1.6), it appears that the relevant ambiguities of the definition of the relative intertwiner $\left(S_{-1, p-1}, T_{-1, p-2} ;\left\{V_{k, p-1-k}\right\}\right.$, $\left.\left\{Z_{k, p-2-k}\right\} ; S_{p-1,-1}, T_{p-2,-1}\right)$, the relative cointertwiner $\left(\Xi^{-1, p}, \Upsilon^{-1, p-1} ;\left\{\Omega^{k, p-1-k}\right\}\right.$, $\left.\left\{\Theta^{k, p-2-k}\right\} ; \Xi^{p,-1}, \Upsilon^{p-1,-1}\right)$ and the relative differential cocycle $\left(\Xi^{p,-1}, \Upsilon^{p-1,-1} ; \Xi^{* p-1,-1}\right.$, $\left.\Upsilon^{* p-2,-1} ; \hat{\Xi}^{p,-1}, \hat{\Upsilon}^{p-1,-1}\right)$ are those parameterized by the relative integer Čech chain $\left(s_{p,-1}\right.$, $\left.t_{p-1,-1}\right)$, the relative real Čech cochain $\left(\xi^{p-1,-1}, v^{p-2,-1}\right)$ and the relative real Čech cochain $\left(\hat{\xi}^{p-1,-1}, \hat{v}^{p-2,-1}\right)$ subject to the condition that the relative coboundary $\left(\delta \hat{\xi}^{p-1,-1}\right.$, $\left.i^{*} \hat{\xi}^{p-1,-1}-\delta \hat{v}^{p-2,-1}\right)$ is integer (cfr. (1.2.8)-(1.2.10), (1.2.20)-(1.2.22) and (1.3.4)-(1.3.6) and the previous discussion). The crucial point to be noted here is that the relative Čech cochain $\left(\xi^{p-1,-1}, v^{p-2,-1}\right)$ parameterizing the ambiguity of the relative Čech cocycle $\left(\Xi^{p,-1}, \Upsilon^{p-1,-1}\right)$ is the same for both the relative cointertwiner and the relative differential cocycle. Taking this into account, from $(2.1 .3),(2.1 .6)$ with $s_{-1, p}=0, t_{-1, p-1}=0$, $\xi^{-1, p-1}=0, v^{-1, p-2}=0$, we find that

$$
\begin{aligned}
\Delta I^{\mathcal{O}}= & (-1)^{p-1}\left[\left\langle S_{p-1,-1}, \hat{\xi}^{p-1,-1}\right\rangle-\left\langle T_{p-2,-1}, \hat{v}^{p-2,-1}\right\rangle\right. \\
& +\left\langle s_{p,-1}, \hat{\Xi}^{p,-1}\right\rangle-\left\langle t_{p-1,-1}, \hat{\Upsilon}^{p-1,-1}\right\rangle \\
& \left.+\left\langle s_{p,-1}, \delta \hat{\xi}^{p-1,-1}\right\rangle-\left\langle t_{p-1,-1}, i^{*} \hat{\xi}^{p-1,-1}-\delta \hat{v}^{p-2,-1}\right\rangle\right] .
\end{aligned}
$$

$\Delta I^{\mathcal{O}}$ is clearly non zero in general. Thus, $I^{\mathcal{O}}$ is not unambiguously defined. However, the above expression suggests that, under certain conditions, $\Delta I^{\mathcal{O}}$ might be integer valued. In such a case, $I^{\mathcal{O}}$ would be unambiguously defined modulo integers.

If $\left(\hat{\xi}^{p-1,-1}, \hat{v}^{p-2,-1}\right)$ were an relative integer Čech cochain, $\Delta I^{\mathcal{O}}$ would be integer. However, because of torsion, the relative Čech cochain $\left(\hat{\xi}^{p-1,-1}, \hat{v}^{p-2,-1}\right)$ is real, being only subject to the condition that the relative coboundary $\left(\delta \hat{\xi}^{p-1,-1}, i^{*} \hat{\xi}^{p-1,-1}-\delta \hat{v}^{p-2,-1}\right)$ is 
integer. So the first two terms of the right hand side of (2.1.8) and, thus, $\Delta I^{\mathcal{O}}$ are generally not integer valued.

If we insist that $\Delta I^{\mathcal{O}}$ be integer, we have to restrict the ambiguity inherent in the choice of the differential cocycle $\left(\Xi^{p,-1}, \Upsilon^{p-1,-1} ; \Xi^{* p-1,-1}, \Upsilon^{* p-2,-1} ; \hat{\Xi}^{p,-1}, \hat{\Upsilon}^{p-1,-1}\right)$ which is responsible for the non integrality of $\left(\hat{\xi}^{p-1,-1}, \hat{v}^{p-2,-1}\right)$. This can be achieved in two steps.

We first restrict the choice of the relative integer Čech cocycle $\left(\hat{\Xi}^{p,-1}, \hat{\Upsilon}^{p-1,-1}\right)$ by fixing its cohomology class in $H_{C \mathbb{Z}}^{p}(X, Y, \mathcal{O})$ among those classes of $H_{C \mathbb{Z}}^{p}(X, Y, \mathcal{O})$ whose image in $H_{C}^{p}(X, Y, \mathcal{O})$ is represented by the relative real Čech cocycle $\left(\Xi^{p,-1}, \Upsilon^{p-1,-1}\right)$ (cfr. subsect. 1.5). By inspecting (1.3.4)-(1.3.6) for given $\left(\xi^{p-1,-1}, v^{p-2,-1}\right)$, it is easy to see that the relative real Cech cochain $\left(\hat{\xi}^{p-1,-1}, \hat{v}^{p-2,-1}\right)$ is restricted in this way to be integer up to a relative real Čech cocycle.

Such a cocycle parameterizes the set of the possible choices of the relative real Cech cochain $\left(\Xi^{* p-1,-1}, \Upsilon^{* p-2,-1}\right)$ for given $\left(\Xi^{p,-1}, \Upsilon^{p-1,-1}\right),\left(\hat{\Xi}^{p,-1}, \hat{\Upsilon}^{p-1,-1}\right)$. It is natural to identify two choices of $\left(\Xi^{* p-1,-1}, \Upsilon^{* p-2,-1}\right)$ if they yield the same value of $I^{\mathcal{O}}$ modulo integers for all $\left(S_{p-1}, T_{p-2}\right) \in Z_{p-1}^{s \mathcal{O}}(X, Y)$. From (2.1.8), on account of (1.2.3), it is apparent that two choices of $\left(\Xi^{* p-1,-1}, \Upsilon^{* p-2,-1}\right)$ are equivalent when their difference is a cohomologically integer relative cocycle (cfr. subsect. 1.5). Thus, the set of equivalence classes of choices of $\left(\Xi^{* p-1,-1}, \Upsilon^{* p-2,-1}\right)$ is parameterized by the quotient $Z_{C}^{p-1}(X, Y, \mathcal{O}) /$ $\tilde{Z}_{C \mathbb{Z}}^{p-1}(X, Y, \mathcal{O})$ or, what is the same, by the relative Cech cohomology torus $H_{C}^{p-1}(X, Y, \mathcal{O}) /$ $\tilde{H}_{C \mathbb{Z}}^{p-1}(X, Y, \mathcal{O})$. From its definition, it is clear that the parametrization is non canonical, depending on an arbitrary choice of a reference relative Cech cochain $\left(\Xi^{* p-1,-1}, \Upsilon^{* p-2,-1}\right)$ corresponding to the origin of the torus.

We next restrict the choice of the relative real Čech cochain $\left(\Xi^{* p-1,-1}, \Upsilon^{* p-2,-1}\right)$ by fixing its image in $H_{C}^{p-1}(X, Y, \mathcal{O}) / \tilde{H}_{C \mathbb{Z}}^{p-1}(X, Y, \mathcal{O})$.

The relative real Cech cochain $\left(\hat{\xi}^{p-1,-1}, \hat{v}^{p-2,-1}\right)$ is finally restricted to be integer up to a cohomologically integer relative cocycle. From (2.1.8), using (1.2.3) again, it follows then that, once the above choices are made, the ambiguity $\Delta I^{\mathcal{O}}$ is integer valued.

Recalling the isomorphisms of singular, de Rham and Čech cohomology discussed in sect. 1.5, we conclude that we can unambiguously define a family of maps $\Phi^{\mathcal{O}}$ : $Z_{p-1}^{s \mathcal{O}}(X, Y) \rightarrow \mathbb{R} / \mathbb{Z}$ by

$$
\Phi^{\mathcal{O}}(S, T ; \Xi, \Upsilon)=I^{\mathcal{O}} \bmod \mathbb{Z}
$$

for $\left(S_{p-1}, T_{p-2}\right) \in Z_{p-1}^{s \mathcal{O}}(X, Y)$, depending on a choice of a relative integer singular cohomology class in $H_{s \mathbb{Z}}^{p}(X, Y)$, a representative $\left(\Xi^{p}, \Upsilon^{p-1}\right) \in Z_{d R \mathbb{Z}}^{p}(X, Y)$ of the image of such 
class in $H_{d R \mathbb{Z}}^{p}(X, Y)$ shown explicitly and a point in the relative de Rham cohomology torus $H_{d R}^{p-1}(X, Y) / H_{d R \mathbb{Z}}^{p-1}(X, Y)$. From (2.1.1), (2.1.4), (2.1.7), (2.1.9), it appears that $\Phi^{\mathcal{O}}$ is $\mathbb{Z}$ linear in the first argument.

When $\left(S_{p-1}, T_{p-2}\right),\left(\Xi^{p}, \Upsilon^{p-1}\right)$ are shifted by amounts given by the right hand sides of (1.2.2), with $s_{-1, p}, t_{-1, p-1} \mathcal{O}$-small, and (1.2.14), respectively, one has

$$
\begin{aligned}
\Delta \Phi^{\mathcal{O}}(S, T ; \Xi, \Upsilon)= & \left\langle S_{-1, p-1}, \xi^{-1, p-1}\right\rangle-\left\langle T_{-1, p-2}, v^{-1, p-2}\right\rangle \\
& +\left\langle s_{-1, p}, \Xi^{-1, p}\right\rangle-\left\langle t_{-1, p-1}, \Upsilon^{-1, p-1}\right\rangle \\
& +\left\langle s_{-1, p}, d \xi^{-1, p-1}\right\rangle-\left\langle t_{-1, p-1}, i^{*} \xi^{-1, p-1}-d v^{-1, p-2}\right\rangle, \quad \bmod \mathbb{Z},
\end{aligned}
$$

as follows readily from (2.1.3).

For reasons explained above, the $H_{d R}^{p-1}(X, Y) / H_{d R \mathbb{Z}}^{p-1}(X, Y)$ parametrization of the maps $\Phi^{\mathcal{O}}$ is not canonical. The changes of the parametrization are in one-to-one correspondence with the shifts in the relative de Rham cohomology torus. By the isomorphisms of de Rham and Čech cohomologies of subsects. 1.4, 1.5, any such shift is represented equivalently by either a relative de Rham cocycle $\left(\Pi^{-1, p-1}, \Sigma^{-1, p-2}\right)$ defined up to cohomologically integer relative de Rham cocycles or a relative real Čech cocycle $\left(\Pi^{p-1,-1}, \Sigma^{p-2,-1}\right)$ defined modulo a cohomologically integer relative real Čech cocycle. The variation of $\Phi^{\mathcal{O}}(S, T ; \Xi, \Upsilon)$ caused by the shift is given by

$$
\Delta \Phi^{\mathcal{O}}(S, T ; \Xi, \Upsilon)=(-1)^{p-1}\left[\left\langle S_{p-1,-1}, \Pi^{p-1,-1}\right\rangle-\left\langle T_{p-2,-1}, \Sigma^{p-2,-1}\right\rangle\right] \bmod \mathbb{Z},
$$

as follows from the first two terms of $(2.1 .8)$ with $\left(\hat{\xi}^{p-1,-1}, \hat{v}^{p-2,-1}\right)$ replaced by $\left(\Pi^{p-1,-1}\right.$, $\left.\Sigma^{p-2,-1}\right)$. It is straightforward to show that

$$
\Delta \Phi^{\mathcal{O}}(S, T ; \Xi, \Upsilon)=-\left[\left\langle S_{-1, p-1}, \Pi^{-1, p-1}\right\rangle-\left\langle T_{-1, p-2}, \Sigma^{-1, p-2}\right\rangle\right]
$$

Indeed, consider the function $I_{1}^{\mathcal{O}}$, eq. (2.1.1). If we vary the Čech-de Rham $p$-cointertwiner $\left(\Xi^{-1, p}, \Upsilon^{-1, p-1} ;\left\{\Omega^{k, p-1-k}\right\},\left\{\Theta^{k, p-2-k}\right\} ; \Xi^{p,-1}, \Upsilon^{p-1,-1}\right)$ by a vanishing amount $\left(\Delta \Xi^{-1, p}\right.$, $\left.\Delta \Upsilon^{-1, p-1} ;\left\{\Delta \Omega^{k, p-1-k}\right\},\left\{\Delta \Theta^{k, p-2-k}\right\} ; \Delta \Xi^{p,-1}, \Delta \Upsilon^{p-1,-1}\right)$, then $\Delta I_{1}^{\mathcal{O}}=0$ trivially. On the other hand, the totally vanishing trivial Cech-de Rham $p$-cointertwiner can be written in the form $(1.2 .20)-(1.2 .22)$ with $\left(\xi^{-1, p-1}, v^{-1, p-2}\right)=\left(\Pi^{-1, p-1}, \Sigma^{-1, p-2}\right),\left(\xi^{p-1,-1}\right.$, 
$\left.v^{p-2,-1}\right)=\left(\Pi^{p-1,-1}, \Sigma^{p-2,-1}\right)$ for suitable Čech-de Rham cochains $\omega^{k, p-2-k}, \theta^{k, p-3-k}$. So, by (2.1.3), $\Delta I_{1}^{\mathcal{O}}=0$ is given by the difference of the two above expressions.

\section{Dependence of $\Phi^{\mathcal{O}}$ on covering choices}

It is important to compare the result of the above construction for two choices of the underlying good open covering of $X, Y, \mathcal{O}(1), \mathcal{O}(2)$. The basic ideas consists in constructing suitable sequences of Čech (co)chains of the covering $\mathcal{O}(1) \cup \mathcal{O}(2)$ (disjoint union) interpolating between the given Čech (co)chains of the individual coverings $\mathcal{O}(1)$, $\mathcal{O}(2)$.

To this end, we explicitly indicate the Čech degree with respect the two coverings. So, $U_{k, l, n}$, say, is a Čech singular chain of Čech degree $k, l$ with respect to $\mathcal{O}(1), \mathcal{O}(2)$, respectively, and dimension $n$. Similarly, $\Lambda^{k, l, n}$, say, is a Čech-de Rham cochain of Čech degree $k, l$ with respect to $\mathcal{O}(1), \mathcal{O}(2)$, respectively, and form degree $n$. Accordingly, we have two operators $\beta_{1}, \beta_{2}$ defined as in (1.1.7) and obeying (1.1.8). Similarly, we have two operators $\delta_{1}, \delta_{2}$ defined as in (1.1.12) and obeying (1.1.13). Further, the pairs $\beta_{1}, \delta_{1}$ and $\beta_{2}, \delta_{2}$ independently satisfy the duality relations (1.1.17). Conversely, we have just one operator $b$ and one operator $d$, which are the same as before and satisfy the duality relations (1.1.16).

A Čech singular chain $U_{k, l, n}$ is a Čech singular chain of $\mathcal{O}(1) \cup \mathcal{O}(2)$ of Čech degree $k+l+1$. A Čech singular chain of the form $U_{k,-1, n}\left(U_{-1, l, n}\right)$ can be identified with a Čech singular chain $U_{k,-1, n}^{(1)}\left(U_{-1, l, n}^{(2)}\right)$ of $\mathcal{O}(1)(\mathcal{O}(2))$ of Čech degree $k(l)$ having the property of being $\mathcal{O}(2)$-small $(\mathcal{O}(1)$-small). The operator $\beta$ appropriate for the Čech singular chains of $\mathcal{O}(1) \cup \mathcal{O}(2)$ is the sum $\beta_{1}+(-1)^{\operatorname{deg}(1)+1} \beta_{2}$ while that for the Čech singular chains of $\mathcal{O}(1)(\mathcal{O}(2))$ is $\beta_{1}\left(\beta_{2}\right)$. Similarly, a Čech-de Rham cochain $\Lambda^{k, l, n}$ is a Čech-de Rham cochain of $\mathcal{O}(1) \cup \mathcal{O}(2)$ of Čech degree $k+l+1$. A Čech-de Rham cochain of the form $\Lambda^{k,-1, n}\left(\Lambda^{-1, l, n}\right)$ can be identified with a Čech-de Rham cochain $\Lambda_{(1)}^{k,-1, n}\left(\Lambda_{(2)}^{-1, l, n}\right)$ of $\mathcal{O}(1)$ $(\mathcal{O}(2))$ of Čech degree $k(l)$. The operator $\delta$ appropriate for the Čech-de Rham cochains of $\mathcal{O}(1) \cup \mathcal{O}(2)$ is the sum $\delta_{1}+(-1)^{\operatorname{deg}(1)+1} \delta_{2}$ while that for the Čech-de Rham cochains of $\mathcal{O}(1)(\mathcal{O}(2))$ is $\delta_{1}\left(\delta_{2}\right)$.

When stating that a sequence of (co)chains forms a relative (co)chain, (co)cycle, (co)boundary, (trivial) (co)intertwiner etc. it is necessary to specify the underlying covering and the relevant $\beta$ or $\delta$ operators. If no label is attached to the (co)chains, it is understood that the covering is $\mathcal{O}(1) \cup \mathcal{O}(2)$ and the $\beta$ or $\delta$ operators are $\beta_{1}+(-1)^{\operatorname{deg}(1)+1} \beta_{2}$, 
$\delta_{1}+(-1)^{\operatorname{deg}(1)+1} \delta_{2}$. If the label $1(2)$ is attached to the (co)chains, it is understood that the covering is $\mathcal{O}(1)(\mathcal{O}(2))$ and the $\beta$ or $\delta$ operators are $\beta_{1}\left(\beta_{2}\right), \delta_{1}\left(\delta_{2}\right)$.

Set $J_{r}=\{(k, l) \mid k, l \in \mathbb{Z}, 0 \leq k, l, 0 \leq k+l \leq r\}, K_{r}=\{k \mid k \in \mathbb{Z}, 0 \leq k \leq r\}, r \in \mathbb{N}$. We say that a sequence of chains $\left(S_{-1,-1, p-1}, T_{-1,-1, p-2} ;\left\{V_{k, l, p-1-k-l} \mid(k, l) \in J_{p-1}\right\}\right.$, $\left.\left\{Z_{k, l, p-2-k-l} \mid(k, l) \in J_{p-2}\right\} ;\left\{S_{k, p-1-k,-1} \mid k \in K_{p-1}\right\},\left\{T_{k, p-2-k,-1} \mid k \in K_{p-2}\right\}\right)$ interpolates two relative Čech singular $p-1$-intertwiners $\left(S_{-1,-1, p-1}^{(1)}, T_{-1,-1, p-2}^{(1)} ;\left\{V_{k,-1, p-1-k}^{(1)} \mid\right.\right.$ $\left.\left.k \in I_{p-1}\right\},\left\{Z_{k,-1, p-2-k}^{(1)} \mid k \in I_{p-2}\right\} ; S_{p-1,-1,-1}^{(1)}, T_{p-2,-1,-1}^{(1)}\right), \quad\left(S_{-1,-1, p-1}^{(2)}, T_{-1,-1, p-2}^{(2)}\right.$; $\left\{V_{-1, k, p-1-k}^{(2)} \mid k \in I_{p-1}\right\},\left\{Z_{-1, k, p-2-k}^{(2)} \mid k \in I_{p-2}\right\} ; S_{-1, p-1,-1}^{(2)}, T_{-1, p-2,-1}^{(2)}$ ) (cfr. eqs. (1.2.5)(1.2.7)), if $S_{-1,-1, p-1}, V_{k, l, p-1-k-l}, S_{k, p-1-k,-1}$ are Čech singular chains of $X, T_{-1,-1, p-2}$, $Z_{k, l, p-2-k-l}, T_{k, p-2-k,-1}$ are Čech singular chains in $Y$ and

$$
\begin{aligned}
& S_{-1,-1, p-1}=\beta_{1} \beta_{2} V_{0,0, p-1}, \\
& T_{-1,-1, p-2}=-\beta_{1} \beta_{2} Z_{0,0, p-2}, \\
& b V_{k, l, p-1-k-l}=\beta_{1} V_{k+1, l, p-2-k-l}+(-1)^{k+1} \beta_{2} V_{k, l+1, p-2-k-l} \\
& +(-1)^{k+l+1} i_{*} Z_{k, l, p-2-k-l}, \quad 0 \leq k, l, \quad 0 \leq k+l \leq p-2, \\
& b Z_{k, l, p-2-k-l}=\beta_{1} Z_{k+1, l, p-3-k-l}+(-1)^{k+1} \beta_{2} Z_{k, l+1, p-3-k-l}, \\
& 0 \leq k, l, \quad 0 \leq k+l \leq p-3, \\
& S_{k, p-1-k,-1}=b V_{k, p-1-k, 0}, \\
& T_{k, p-2-k,-1}=-(-1)^{p-1} b Z_{k, p-2-k, 0},
\end{aligned}
$$

with

$$
\begin{aligned}
& S_{-1,-1, p-1}^{(1)}=S_{-1,-1, p-1}, \quad S_{-1,-1, p-1}^{(2)}=S_{-1,-1, p-1}, \\
& T_{-1,-1, p-2}^{(1)}=T_{-1,-1, p-2}, \quad T_{-1,-1, p-2}^{(2)}=T_{-1,-1, p-2}, \\
& V_{k,-1, p-1-k}^{(1)}=\beta_{2} V_{k, 0, p-1-k}, \quad V_{-1, k, p-1-k}^{(2)}=(-1)^{k} \beta_{1} V_{0, k, p-1-k}, \\
& \quad 0 \leq k \leq p-1,
\end{aligned}
$$




$$
\begin{aligned}
& Z_{k,-1, p-2-k}^{(1)}=-\beta_{2} Z_{k, 0, p-2-k}, \quad Z_{-1, k, p-2-k}^{(2)}=-(-1)^{k} \beta_{1} Z_{0, k, p-2-k}, \\
& \quad 0 \leq k \leq p-2 \\
& S_{p-1,-1,-1}^{(1)}=\beta_{2} S_{p-1,0,-1}, \quad S_{-1, p-1,-1}^{(2)}=(-1)^{p-1} \beta_{1} S_{0, p-1,-1}, \\
& T_{p-2,-1,-1}^{(1)}=\beta_{2} T_{p-2,0,-1}, \quad T_{-1, p-2,-1}^{(2)}=(-1)^{p-2} \beta_{1} T_{0, p-2,-1} .
\end{aligned}
$$

It is straightforward to check that the above relations are compatible with the relations (1.2.5)-(1.2.7) obeyed by the relative Čech singular intertwiners.

For $r \in \mathbb{N}$, define $J^{r}=\{(k, l) \mid k, l \in \mathbb{Z},-1 \leq k, l,-1 \leq k+l \leq r\}, K^{r}=\{k \mid k \in$ $\mathbb{Z},-1 \leq k \leq r\}$. We say that a sequence of cochains $\left(\Xi^{-1,-1, p}, \Upsilon^{-1,-1, p-1} ;\left\{\Omega^{k, l, p-2-k-l} \mid\right.\right.$ $\left.(k, l) \in J^{p-2}\right\},\left\{\Theta^{k, l, p-3-k-l} \mid(k, l) \in J^{p-3}\right\} ;\left\{\Xi^{k, p-1-k,-1} \mid k \in K^{p}\right\}, \quad\left\{\Upsilon^{k, p-2-k,-1} \mid k \in\right.$ $\left.\left.K^{p-1}\right\}\right)$ interpolates two relative Čech-de Rham $p$-cointertwiners $\left(\Xi_{(1)}^{-1,-1, p}, \Upsilon_{(1)}^{-1,-1, p-1}\right.$; $\left.\left\{\Omega_{(1)}^{k,-1, p-1-k} \mid k \in I^{p-1}\right\}, \quad\left\{\Theta_{(1)}^{k,-1, p-2-k} \mid k \in I^{p-2}\right\} ; \Xi_{(1)}^{p,-1,-1}, \Upsilon_{(1)}^{p-1,-1,-1}\right), \quad\left(\Xi_{(2)}^{-1,-1, p}\right.$, $\Upsilon_{(2)}^{-1,-1, p-1} ;\left\{\Omega_{(2)}^{-1, k, p-1-k} \mid k \in I^{p-1}\right\},\left\{\Theta_{(2)}^{-1, k, p-2-k} \mid k \in I^{p-2}\right\} ; \Xi_{(2)}^{-1, p,-1}, \Upsilon_{(2)}^{-1, p-1,-1}$ ) (cfr. eqs. (1.2.17)-(1.2.19)), if $\Xi^{-1,-1, p-1}, \Omega^{k, l, p-1-k-l}, \Xi^{k, p-1-k,-1}$ are Čech-de Rham cochains of $X, \Upsilon^{-1,-1, p-2}, \Theta^{k, l, p-2-k-l}, \Upsilon^{k, p-2-k,-1}$ are Čech-de Rham cochains in $Y$ and

$$
\begin{aligned}
& \delta_{1} \Xi^{-1,-1, p}=d \Omega^{0,-1, p-1}, \\
& \delta_{2} \Xi^{-1,-1, p}=d \Omega^{-1,0, p-1} \\
& \delta_{1} \Upsilon^{-1,-1, p-1}=-d \Theta^{0,-1, p-2}+i^{*} \Omega^{0,-1, p-1}, \\
& \delta_{2} \Upsilon^{-1,-1, p-1}=-d \Theta^{-1,0, p-2}+i^{*} \Omega^{-1,0, p-1}, \\
& d \Omega^{k, l, p-2-k-l}=\delta_{1} \Omega^{k-1, l, p-1-k-l}+(-1)^{k+1} \delta_{2} \Omega^{k, l-1, p-1-k-l}, \\
& \quad-1 \leq k, l, \quad 0 \leq k+l \leq p-2, \\
& d \Theta^{k, l, p-3-k-l}=\delta_{1} \Theta^{k-1, l, p-2-k-l}+(-1)^{k+1} \delta_{2} \Theta^{k, l-1, p-2-k-l} \\
& +(-1)^{k+l+1} i^{*} \Omega^{k, l, p-2-k-l}, \quad-1 \leq k, l, \quad 0 \leq k+l \leq p-3, \\
& d \Xi^{k, p-1-k,-1}=\delta_{1} \Omega^{k-1, p-1-k, 0}+(-1)^{k+1} \delta_{2} \Omega^{k, p-2-k, 0},
\end{aligned}
$$




$$
\begin{aligned}
& d \Upsilon^{k, p-2-k,-1}=(-1)^{p-1}\left(\delta_{1} \Theta^{k-1, p-2-k, 0}+(-1)^{k+1} \delta_{2} \Theta^{k, p-3-k, 0}\right. \\
& \left.+(-1)^{p-1} i^{*} \Omega^{k, p-2-k, 0}\right)
\end{aligned}
$$

with

$$
\begin{aligned}
& \Xi_{(1)}^{-1,-1, p}=\Xi^{-1,-1, p}, \quad \Xi_{(2)}^{-1,-1, p}=\Xi^{-1,-1, p} \\
& \Upsilon_{(1)}^{-1,-1, p-1}=\Upsilon^{-1,-1, p-1}, \quad \Upsilon_{(2)}^{-1,-1, p-1}=\Upsilon^{-1,-1, p-1}, \\
& \Omega_{(1)}^{k,-1, p-1-k}=\Omega^{k,-1, p-1-k}, \quad \Omega_{(2)}^{-1, k, p-1-k}=\Omega^{-1, k, p-1-k}, \quad 0 \leq k \leq p-1 \\
& \Theta_{(1)}^{k,-1, p-2-k}=\Theta^{k,-1, p-2-k}, \quad \Theta_{(2)}^{-1, k, p-2-k}=\Theta^{-1, k, p-2-k}, \quad 0 \leq k \leq p-2, \\
& \Xi_{(1)}^{p,-1,-1}=\Xi^{p,-1,-1}, \quad \Xi_{(2)}^{-1, p,-1}=\Xi^{-1, p,-1}, \\
& \Upsilon_{(1)}^{p-1,-1,-1}=\Upsilon^{p-1,-1,-1}, \quad \Upsilon_{(2)}^{-1, p-1,-1}=\Upsilon^{-1, p-1,-1}
\end{aligned}
$$

1. It is straightforward to check that the above relations are compatible with the relations (1.2.17)-(1.2.19) obeyed by the relative Čech-de Rham cointertwiners.

Using the interpolating sequences of (co)chains introduced above, one defines for $0 \leq$ $k \leq p-2$

$$
\begin{aligned}
S_{1 k} & =\sum_{l=0}^{k}\left\langle V_{l, k-l, p-1-k}, \delta_{1} \Omega^{l-1, k-l, p-1-k}+(-1)^{l+1} \delta_{2} \Omega^{l, k-l-1, p-1-k}\right\rangle \\
& +\sum_{l=0}^{k}\left\langle Z_{l, k-l, p-2-k}, \delta_{1} \Theta^{l-1, k-l, p-2-k}+(-1)^{l+1} \delta_{2} \Theta^{l, k-l-1, p-2-k}\right\rangle .
\end{aligned}
$$

Using the relations (1.1.16), (1.1.17), (2.2.2), (2.2.8), (2.2.5), (2.2.11), one finds that, for $1 \leq k \leq p-2$

$$
\begin{aligned}
& (-1)^{k}\left\langle V_{k,-1, p-1-k}^{(1)}, \Omega_{(1)}^{k,-1, p-1-k}\right\rangle-(-1)^{k}\left\langle V_{-1, k, p-1-k}^{(2)}, \Omega_{(2)}^{-1, k, p-1-k}\right\rangle \\
& -(-1)^{k}\left\langle Z_{k,-1, p-2-k}^{(1)}, \Theta_{(1)}^{k,-1, p-2-k}\right\rangle+(-1)^{k}\left\langle Z_{-1, k, p-2-k}^{(2)}, \Theta_{(2)}^{-1, k, p-2-k}\right\rangle \\
& +S_{1 k}-S_{1 k-1}=0 .
\end{aligned}
$$

1 In these formulae, it is assumed conventionally that any Cech-de Rham cochain $\lambda^{k, l, m}=0$ whenever $k, l$ do not satisfy the restrictions listed at the beginning of this paragraph. 
Hence,

$$
\begin{aligned}
& \sum_{k=1}^{p-2}(-1)^{k}\left\langle V_{k,-1, p-1-k}^{(1)}, \Omega_{(1)}^{k,-1, p-1-k}\right\rangle-\sum_{k=1}^{p-2}(-1)^{k}\left\langle Z_{k,-1, p-2-k}^{(1)}, \Theta_{(1)}^{k,-1, p-2-k}\right\rangle \\
- & \sum_{k=1}^{p-2}(-1)^{k}\left\langle V_{-1, k, p-1-k}^{(2)}, \Omega_{(2)}^{-1, k, p-1-k}\right\rangle+\sum_{k=1}^{p-2}(-1)^{k}\left\langle Z_{-1, k, p-2-k}^{(2)}, \Theta_{(2)}^{-1, k, p-2-k}\right\rangle \\
+ & S_{1 p-2}-S_{10}=0 .
\end{aligned}
$$

From the definition (2.2.13), using (1.1.17), (2.2.5), (2.2.11) with $k=0$, one easily sees that

$$
\begin{aligned}
S_{10}= & -\left\langle V_{0,-1, p-1}^{(1)}, \Omega_{(1)}^{0,-1, p-1}\right\rangle+\left\langle Z_{0,-1, p-2}^{(1)}, \Theta_{(1)}^{0,-1, p-2}\right\rangle \\
& +\left\langle V_{-1,0, p-1}^{(2)}, \Omega_{(2)}^{-1,0, p-1}\right\rangle-\left\langle Z_{-1,0, p-2}^{(2)}, \Theta_{(2)}^{-1,0, p-2}\right\rangle .
\end{aligned}
$$

Further, from the definition (2.2.13), using (1.1.16), (1.1.17), (2.2.2a), (2.2.5a), (2.2.8), (2.2.11a), one finds

$$
\begin{aligned}
S_{1 p-2} & =\sum_{k=0}^{p-1}\left\langle V_{k, p-1-k, 0}, \delta_{1} \Omega^{k-1, p-1-k, 0}+(-1)^{k+1} \delta_{2} \Omega^{k, p-2-k, 0}\right\rangle \\
& +\sum_{k=0}^{p-2}\left\langle Z_{k, p-2-k, 0}, \delta_{1} \Theta^{k-1, p-2-k, 0}+(-1)^{k+1} \delta_{2} \Theta^{k, p-3-k, 0}+(-1)^{p-1} i^{*} \Omega^{k, p-2-k, 0}\right\rangle \\
& +(-1)^{p-1}\left\langle V_{p-1,-1,0}^{(1)}, \Omega_{(1)}^{p-1,-1,0}\right\rangle-(-1)^{p-1}\left\langle V_{-1, p-1,0}^{(2)}, \Omega_{(2)}^{-1, p-1,0}\right\rangle .
\end{aligned}
$$

Let $I_{1}^{\mathcal{O}(1)}\left(I_{1}^{\mathcal{O}(2)}\right)$ be constructed according (2.1.1) using the above (co)intertwiners marked by the label 1 (2). Substituting (2.2.16) and (2.2.17) into (2.2.15) and using (2.2.3), (2.2.9), one finds

$$
I_{1}^{\mathcal{O}(2)}-I_{1}^{\mathcal{O}(1)}=\sum_{k=0}^{p-1}\left\langle S_{k, p-1-k,-1}, \Xi^{k, p-1-k,-1}\right\rangle-\sum_{k=0}^{p-2}\left\langle T_{k, p-2-k,-1}, \Upsilon^{k, p-2-k,-1}\right\rangle
$$

We say that a sequence of chains $\left(\left\{S_{k, p-1-k,-1} \mid k \in K_{p-1}\right\},\left\{T_{k, p-2-k,-1} \mid k \in K_{p-2}\right\}\right)$ interpolates two relative integer Čech $p-1$-cycles $\left(S_{p-1,-1,-1}^{(1)}, T_{p-2,-1,-1}^{(1)}\right), \quad\left(S_{-1, p-1,-1}^{(2)}\right.$, $T_{-1, p-2,-1}^{(2)}$ ) (cfr. eqs. (1.2.3)), if $S_{k, p-1-k,-1}$ are integer Čech chains of $X, T_{k, p-2-k,-1}$ 
are integer Čech chains in $Y$ and

$$
\begin{aligned}
& \beta_{1} S_{k+1, p-2-k,-1}+(-1)^{k+1} \beta_{2} S_{k, p-1-k,-1}-i_{*} T_{k, p-2-k,-1}=0, \\
& 0 \leq k \leq p-2, \\
& -\beta_{1} T_{k+1, p-3-k,-1}-(-1)^{k+1} \beta_{2} T_{k, p-2-k,-1}=0, \quad 0 \leq k \leq p-3,
\end{aligned}
$$

with

$$
\begin{aligned}
& S_{p-1,-1,-1}^{(1)}=\beta_{2} S_{p-1,0,-1}, \quad S_{-1, p-1,-1}^{(2)}=(-1)^{p-1} \beta_{1} S_{0, p-1,-1}, \\
& T_{p-2,-1,-1}^{(1)}=\beta_{2} T_{p-2,0,-1}, \quad T_{-1, p-2,-1}^{(2)}=(-1)^{p-2} \beta_{1} T_{0, p-2,-1} .
\end{aligned}
$$

The above relations are compatible with the relations (1.2.3) obeyed by the integer Čech cycles.

We say that a sequence of cochains $\left(\left\{\Xi^{k, p-1-k,-1} \mid k \in K^{p}\right\},\left\{\Upsilon^{k, p-2-k,-1} \mid k \in K^{p-1}\right\}\right.$; $\left\{\Xi^{* k, p-2-k,-1} \mid k \in K^{p-1}\right\},\left\{\Upsilon^{* k, p-3-k,-1} \mid k \in K^{p-2}\right\} ;\left\{\hat{\Xi}^{k, p-1-k,-1} \mid k \in K^{p}\right\},\left\{\hat{\Upsilon}^{k, p-2-k,-1} \mid\right.$ $\left.\left.k \in K^{p-1}\right\}\right)$ interpolates two given relative differential $p$-cocycles $\left(\Xi_{(1)}^{p,-1,-1}, \Upsilon_{(1)}^{p-1,-1,-1}\right.$; $\left.\Xi_{(1)}^{* p-1,-1,-1}, \Upsilon_{(1)}^{* p-2,-1,-1} ; \hat{\Xi}_{(1)}^{p,-1,-1}, \hat{\Upsilon}_{(1)}^{p-1,-1,-1}\right), \quad\left(\Xi_{(2)}^{-1, p,-1}, \Upsilon_{(2)}^{-1, p-1,-1} ; \Xi_{(2)}^{*-1, p-1,-1}\right.$, $\Upsilon_{(2)}^{*-1, p-2,-1} ; \hat{\Xi}_{(2)}^{-1, p,-1}, \hat{\Upsilon}_{(2)}^{-1, p-1,-1}$ ) (cfr. eqs. (1.3.1)-(1.3.3)), if $\Xi^{k, p-1-k,-1}, \Xi^{* k, p-2-k,-1}$ are real Čech cochains of $X, \hat{\Xi}^{k, p-1-k,-1}$ is an integer Čech cochain of $X, \Upsilon^{k, p-2-k,-1}$, $\Upsilon^{* k, p-3-k,-1}$ are real Čech cochains of $Y, \hat{\Upsilon}^{k, p-2-k,-1}$ is an integer Čech cochain of $Y$ such that

$$
\begin{aligned}
& \delta_{1} \Xi^{k-1, p-k,-1}+(-1)^{k+1} \delta_{2} \Xi^{k, p-1-k,-1}=0, \quad-1 \leq k \leq p+1 \\
& i^{*} \Xi^{k, p-1-k,-1}-\delta_{1} \Upsilon^{k-1, p-1-k,-1}-(-1)^{k+1} \delta_{2} \Upsilon^{k, p-2-k,-1}=0 \\
& \quad-1 \leq k \leq p \\
& \delta_{1} \Xi^{* k-1, p-1-k,-1}+(-1)^{k+1} \delta_{2} \Xi^{* k, p-2-k,-1}=\hat{\Xi}^{k, p-1-k,-1}-\Xi^{k, p-1-k,-1}, \\
& \quad-1 \leq k \leq p \\
& i^{*} \Xi^{* k, p-2-k,-1}-\delta_{1} \Upsilon^{* k-1, p-2-k,-1}-(-1)^{k+1} \delta_{2} \Upsilon^{* k, p-3-k,-1}
\end{aligned}
$$




$$
\begin{aligned}
& =\hat{\Upsilon}^{k, p-2-k,-1}-\Upsilon^{k, p-2-k,-1}, \quad-1 \leq k \leq p-1, \\
& \delta_{1} \hat{\Xi}^{k-1, p-k,-1}+(-1)^{k+1} \delta_{2} \hat{\Xi}^{k, p-1-k,-1}=0, \quad-1 \leq k \leq p+1, \\
& i^{*} \hat{\Xi}^{k, p-1-k,-1}-\delta_{1} \hat{\Upsilon}^{k-1, p-1-k,-1}-(-1)^{k+1} \delta_{2} \hat{\Upsilon}^{k, p-2-k,-1}=0, \\
& \quad-1 \leq k \leq p,
\end{aligned}
$$

with

$$
\begin{aligned}
& \Xi_{(1)}^{p,-1,-1}=\Xi^{p,-1,-1}, \quad \Xi_{(2)}^{-1, p,-1}=\Xi^{-1, p,-1}, \\
& \Upsilon_{(1)}^{p-1,-1,-1}=\Upsilon^{p-1,-1,-1}, \quad \Upsilon_{(2)}^{-1, p-1,-1}=\Upsilon^{-1, p-1,-1}, \\
& \Xi_{(1)}^{* p-1,-1,-1}=\Xi^{* p-1,-1,-1}, \quad \Xi_{(2)}^{*-1, p-1,-1}=\Xi^{*-1, p-1,-1}, \\
& \Upsilon_{(1)}^{* p-2,-1,-1}=\Upsilon^{* p-2,-1,-1}, \quad \Upsilon_{(2)}^{*-1, p-2,-1}=\Upsilon^{*-1, p-2,-1}, \\
& \hat{\Xi}_{(1)}^{p,-1,-1}=\hat{\Xi}^{p,-1,-1}, \quad \hat{\Xi}_{(2)}^{-1, p,-1}=\hat{\Xi}^{-1, p,-1}, \\
& \hat{\Upsilon}_{(1)}^{p-1,-1,-1}=\hat{\Upsilon}^{p-1,-1,-1}, \quad \hat{\Upsilon}_{(2)}^{-1, p-1,-1}=\hat{\Upsilon}^{-1, p-1,-1}
\end{aligned}
$$

(cfr. footnote 1). It is straightforward to check that the above relations are compatible with the relations (1.3.1)-(1.3.3) obeyed by the relative differential cocycles.

Using the interpolating sequences of (co)chains just introduced, one defines

$$
\begin{aligned}
S_{2} & =\sum_{k=0}^{p-1}\left\langle S_{k, p-1-k,-1}, \delta_{1} \Xi^{* k-1, p-1-k,-1}+(-1)^{k+1} \delta_{2} \Xi^{* k, p-2-k,-1}\right\rangle \\
& -\sum_{k=0}^{p-2}\left\langle T_{k, p-2-k,-1}, i^{*} \Xi^{* k, p-2-k,-1}-\delta_{1} \Upsilon^{* k-1, p-2-k,-1}-(-1)^{k+1} \delta_{2} \Upsilon^{* k, p-3-k,-1}\right\rangle .
\end{aligned}
$$

Using (2.2.22), one has immediately

$$
\begin{aligned}
S_{2}= & -\sum_{k=0}^{p-1}\left\langle S_{k, p-1-k,-1}, \Xi^{k, p-1-k,-1}\right\rangle+\sum_{k=0}^{p-2}\left\langle T_{k, p-2-k,-1}, \Upsilon^{k, p-2-k,-1}\right\rangle \\
& +\sum_{k=0}^{p-1}\left\langle S_{k, p-1-k,-1}, \hat{\Xi}^{k, p-1-k,-1}\right\rangle-\sum_{k=0}^{p-2}\left\langle T_{k, p-2-k,-1}, \hat{\Upsilon}^{k, p-2-k,-1}\right\rangle
\end{aligned}
$$


On the other hand, using (1.1.17), (2.2.19), (2.2.20), (2.2.25), one finds

$$
\begin{aligned}
S_{2}=(-1)^{p-1} & {\left[\left\langle S_{-1, p-1,-1}^{(2)}, \Xi_{(2)}^{*-1, p-1,-1}\right\rangle-\left\langle T_{-1, p-2,-1}^{(2)}, \Upsilon_{(2)}^{*-1, p-2,-1}\right\rangle\right.} \\
& \left.-\left\langle S_{p-1,-1,-1}^{(1)}, \Xi_{(1)}^{* p-1,-1,-1}\right\rangle+\left\langle T_{p-2,-1,-1}^{(1)}, \Upsilon_{(1)}^{* p-2,-1,-1}\right\rangle\right] .
\end{aligned}
$$

Now, we note that

$$
\sum_{k=0}^{p-1}\left\langle S_{k, p-1-k,-1}, \hat{\Xi}^{k, p-1-k,-1}\right\rangle-\sum_{k=0}^{p-2}\left\langle T_{k, p-2-k,-1}, \hat{\Upsilon}^{k, p-2-k,-1}\right\rangle=0 \bmod \mathbb{Z}
$$

Let $I_{2}^{\mathcal{O}(1)}\left(I_{2}^{\mathcal{O}(2)}\right)$ be constructed according (2.1.4) using the integer Čech cycle and the differential cocycle marked by the label 1 (2). From (2.2.28), (2.2.29), one has then

$$
I_{2}^{\mathcal{O}(2)}-I_{2}^{\mathcal{O}(1)}=-\sum_{k=0}^{p-1}\left\langle S_{k, p-1-k,-1}, \Xi^{k, p-1-k,-1}\right\rangle+\sum_{k=0}^{p-2}\left\langle T_{k, p-2-k,-1}, \Upsilon^{k, p-2-k,-1}\right\rangle \quad \bmod \mathbb{Z}
$$

Let $\left(S_{p-1}, T_{p-2}\right) \in Z_{p-1}^{s \mathcal{O}(i)}(X, Y),\left(\Xi^{p}, \Upsilon^{p-1}\right) \in Z_{d R \mathbb{Z}}^{p}(X, Y)$ be respectively an $\mathcal{O}(i)-$ small relative singular cycle, $i=1,2$, (cfr. eq. (1.2.1) and subsect. 1.2) and a cohomologically integer relative de Rham cocycle (cfr. eq. (1.2.13) and subsect. 1.5). Let us now repeat the construction described in sect. 2.1 individually for each of the covering $\mathcal{O}(i)$.

Then, the relative singular cycles $\left(S_{-1,-1, p-1}^{(1)}, T_{-1,-1, p-2}^{(1)}\right)=\left(S_{-1,-1, p-1}, T_{-1,-1, p-2}\right)$, $\left(S_{-1,-1, p-1}^{(2)}, T_{-1,-1, p-2}^{(2)}\right)=\left(S_{-1,-1, p-1}, T_{-1,-1, p-2}\right)$ extend to Čech singular intertwiners $\left(S_{-1,-1, p-1}^{(1)}, T_{-1,-1, p-2}^{(1)} ;\left\{V_{k,-1, p-1-k}^{(1)}\right\},\left\{Z_{k,-1, p-2-k}^{(1)}\right\} ; S_{p-1,-1,-1}^{(1)}, T_{p-2,-1,-1}^{(1)}\right),\left(S_{-1,-1, p-1}^{(2)}\right.$, $\left.T_{-1,-1, p-2}^{(2)} ;\left\{V_{-1, k, p-1-k}^{(2)}\right\},\left\{Z_{-1, k, p-2-k}^{(2)}\right\} ; S_{-1, p-1,-1}^{(2)}, T_{-1, p-2,-1}^{(2)}\right)$ defined up to shifts by trivial intertwiners leaving $\left(S_{-1,-1, p-1}^{(1)}, T_{-1,-1, p-2}^{(1)}\right),\left(S_{-1,-1, p-1}^{(2)}, T_{-1,-1, p-2}^{(2)}\right)$ unchanged, respectively, (cfr. subsects. 1.4 and 2.1).

In similar fashion, the relative de Rham cocycles $\left(\Xi_{(1)}^{-1,-1, p}, \Upsilon_{(1)}^{-1,-1, p-1}\right)=\left(\Xi^{-1,-1, p}\right.$, $\left.\Upsilon^{-1,-1, p-1}\right), \quad\left(\Xi_{(2)}^{-1,-1, p}, \Upsilon_{(2)}^{-1,-1, p-1}\right)=\left(\Xi^{-1,-1, p}, \Upsilon^{-1,-1, p-1}\right)$ extend to Čech-de Rham cointertwiners $\left(\Xi_{(1)}^{-1,-1, p}, \Upsilon_{(1)}^{-1,-1, p-1} ;\left\{\Omega_{(1)}^{k,-1, p-1-k}\right\},\left\{\Theta_{(1)}^{k,-1, p-2-k}\right\} ; \Xi_{(1)}^{p,-1,-1}, \Upsilon_{(1)}^{p-1,-1,-1}\right)$, $\left(\Xi_{(2)}^{-1,-1, p}, \Upsilon_{(2)}^{-1,-1, p-1} ;\left\{\Omega_{(2)}^{-1, k, p-1-k}\right\},\left\{\Theta_{(2)}^{-1, k, p-2-k}\right\} ; \Xi_{(2)}^{-1, p,-1}, \Upsilon_{(2)}^{-1, p-1,-1}\right)$ defined up to shifts by trivial cointertwiners leaving $\left(\Xi_{(1)}^{-1,-1, p}, \Upsilon_{(1)}^{-1,-1, p-1}\right),\left(\Xi_{(2)}^{-1,-1, p}, \Upsilon_{(2)}^{-1,-1, p-1}\right)$ unchanged, respectively, (cfr. subsects. 1.4 and 2.1). In turn, the cohomologically integer real Čech cocycles $\left(\Xi_{(1)}^{p,-1,-1}, \Upsilon_{(1)}^{p-1,-1,-1}\right),\left(\Xi_{(2)}^{-1, p,-1}, \Upsilon_{(2)}^{-1, p-1,-1}\right)$ so obtained extend to relative differential cocycles $\left(\Xi_{(1)}^{p,-1,-1}, \Upsilon_{(1)}^{p-1,-1,-1} ; \Xi_{(1)}^{* p-1,-1,-1}, \Upsilon_{(1)}^{* p-2,-1,-1} ; \hat{\Xi}_{(1)}^{p,-1,-1}, \hat{\Upsilon}_{(1)}^{p-1,-1,-1}\right)$, $\left(\Xi_{(2)}^{-1, p,-1}, \Upsilon_{(2)}^{-1, p-1,-1} ; \Xi_{(2)}^{*-1, p-1,-1}, \Upsilon_{(2)}^{*-1, p-2,-1} ; \hat{\Xi}_{(2)}^{-1, p,-1}, \hat{\Upsilon}_{(2)}^{-1, p-1,-1}\right)$, defined up to shifts 
by torsion differential cocycles leaving $\left(\Xi_{(1)}^{p,-1,-1}, \Upsilon_{(1)}^{p-1,-1,-1}\right),\left(\Xi_{(2)}^{-1, p,-1}, \Upsilon_{(2)}^{-1, p-1,-1}\right)$ unchanged, respectively, (cfr. subsects. 1.5 and 2.1).

Using the above two sets of relative data, we can compute $I^{\mathcal{O}(i)}, i=1,2$, using (2.1.7). Since the choice of the relative data is not unique, $I^{\mathcal{O}(i)}$ is affected by an indetermination $\Delta I^{\mathcal{O}(i)}$ given by $(2.1 .8)$.

Next, our aim is to evaluate the difference $I^{\mathcal{O}(2)}-I^{\mathcal{O}(1)}$ modulo integers by constructing suitable interpolating sequences between the relative data of the two coverings involved and exploiting the results $(2.2 .18),(2.2 .31)$. In order to do that, we have first to find out under which conditions such sequences do indeed exist.

Let us assume that $\left(S_{-1,-1, p-1}^{(1)}, T_{-1,-1, p-2}^{(1)} ;\left\{V_{k,-1, p-1-k}^{(1)}\right\},\left\{Z_{k,-1, p-2-k}^{(1)}\right\} ; S_{p-1,-1,-1}^{(1)}\right.$, $\left.T_{p-2,-1,-1}^{(1)}\right),\left(S_{-1,-1, p-1}^{(2)}, T_{-1,-1, p-2}^{(2)} ;\left\{V_{-1, k, p-1-k}^{(2)}\right\},\left\{Z_{-1, k, p-2-k}^{(2)}\right\} ; S_{-1, p-1,-1}^{(2)}, T_{-1, p-2,-1}^{(2)}\right)$ are two relative Čech singular $p-1$-intertwiners (cfr. eqs. (1.2.5)-(1.2.7)) such that $\left(S_{-1,-1, p-1}^{(1)}, T_{-1,-1, p-2}^{(1)}\right)=\left(S_{-1,-1, p-1}^{(2)}, T_{-1,-1, p-2}^{(2)}\right)$. Then, after possibly shifting the intertwiners by trivial intertwiners (cfr. eqs. (1.2.8)-(1.2.10)) preserving this condition, there exists a sequence of chains $\left(S_{-1,-1, p-1}, T_{-1,-1, p-2} ;\left\{V_{k, l, p-1-k-l}\right\},\left\{Z_{k, l, p-2-k-l}\right\}\right.$; $\left.\left\{S_{k, p-1-k,-1}\right\},\left\{T_{k, p-2-k,-1}\right\}\right)$ interpolating the intertwiners, i.e satisfying (2.2.1)-(2.2.6).

Here is a sketch of the proof. We begin with noting that, if $U_{k, l, n}$ is a Cech singular chain with $n>-1$ such that $\beta_{1} \beta_{2} U_{k, l, n}=0$, then there are Čech singular chains $U_{k+1, l, n}$, $U_{k, l+1, n}$ such that $U_{k, l, n}=\beta_{1} U_{k+1, l, n}+(-1)^{k+1} \beta_{2} U_{k, l+1, n}$. This follows from the triviality of the $\beta_{1}, \beta_{2}$ homology for $\mathcal{O}(1), \mathcal{O}(2)$-small chains, respectively, when $n>-1$ and the fact that, if either $k<-1$ or $l<-1$, then $V_{k, l, n}=0$ for any Čech singular chains $V_{k, l, n}$. Set

$$
\begin{aligned}
& S_{-1,-1, p-1}=S_{-1,-1, p-1}^{(1)}=S_{-1,-1, p-1}^{(2)}, \\
& T_{-1,-1, p-2}=T_{-1,-1, p-2}^{(1)}=T_{-1,-1, p-2}^{(2)} .
\end{aligned}
$$

Then, $\left(S_{-1,-1, p-1}, T_{-1,-1, p-2}\right)$ is a relative singular $p-1-$ cycle,

$$
\begin{aligned}
& b S_{-1,-1, p-1}-i_{*} T_{-1,-1, p-2}=0 \\
& -b T_{-1,-1, p-2}=0 .
\end{aligned}
$$

Hence, there is a chain $V_{0,0, p-1}$ of $X$ and a chain $Z_{0,0, p-2}$ of $Y$ satisfying (2.2.1). By substituting (2.2.1) into (2.2.33), one finds that (2.2.2) holds for $k, l=0$ for some chains 
$V_{1,0, p-2}, V_{0,1, p-2}$ of $X$ and $Z_{1,0, p-3}, Z_{0,1, p-3}$ of $Y$. The proof of (2.2.2) is completed by a straightforward induction on the value of $k+l . S_{k, p-1-k,-1}, T_{k, p-2-k,-1}$ are then defined according (2.2.3). Next, one verifies that relations (2.2.4)-(2.2.6) define two relative Čech singular $p-1$-intertwiners extending $\left(S_{-1,-1, p-1}^{(1)}, T_{-1,-1, p-2}^{(1)}\right),\left(S_{-1,-1, p-1}^{(2)}, T_{-1,-1, p-2}^{(2)}\right)$. Thus, these intertwiners must equal the original intertwiners up to trivial shifts preserving $\left(S_{-1,-1, p-1}^{(1)}, T_{-1,-1, p-2}^{(1)}\right),\left(S_{-1,-1, p-1}^{(2)}, T_{-1,-1, p-2}^{(2)}\right)$ (see the discussion of subsect. 1.4).

The sequence of chains $\left(\left\{S_{k, p-1-k,-1}\right\},\left\{T_{k, p-2-k,-1}\right\}\right)$ interpolates the integer Cech cycles $\left(S_{p-1,-1,-1}^{(1)}, T_{p-2,-1,-1}^{(1)}\right),\left(S_{-1, p-1,-1}^{(2)}, T_{-1, p-2,-1}^{(2)}\right)$, i.e. it satisfies $(2.2 .19)-(2.2 .20)$. These statements are straightforwardly verified.

Assume that $\mathcal{O}(1) \cup \mathcal{O}(2)$ is a good covering of the pair $X, Y$ and that $\left(\Xi_{(1)}^{-1,-1, p}\right.$, $\left.\Upsilon_{(1)}^{-1,-1, p-1} ;\left\{\Omega_{(1)}^{k,-1, p-1-k}\right\},\left\{\Theta_{(1)}^{k,-1, p-2-k}\right\} ; \Xi_{(1)}^{p,-1,-1}, \Upsilon_{(1)}^{p-1,-1,-1}\right), \quad\left(\Xi_{(2)}^{-1,-1, p}, \Upsilon_{(2)}^{-1,-1, p-1}\right.$; $\left.\left\{\Omega_{(2)}^{-1, k, p-1-k}\right\},\left\{\Theta_{(2)}^{-1, k, p-2-k}\right\} ; \Xi_{(2)}^{-1, p,-1}, \Upsilon_{(2)}^{-1, p-1,-1}\right)$ are two relative Čech-de Rham $p$-cointertwiners (cfr. eqs. (1.2.17)-(1.2.19)) such that $\left(\Xi_{(1)}^{-1,-1, p}, \Upsilon_{(1)}^{-1,-1, p-1}\right)=\left(\Xi_{(2)}^{-1,-1, p}\right.$, $\left.\Upsilon_{(2)}^{-1,-1, p-1}\right)$. Then, after possibly shifting the cointertwiners by trivial cointertwiners (cfr. eqs. (1.2.20)-(1.2.22)) preserving this condition, there exists a sequence of cochains $\left(\Xi^{-1,-1, p}, \Upsilon^{-1,-1, p-1} ; \quad\left\{\Omega^{k, l, p-2-k-l}\right\},\left\{\Theta^{k, l, p-3-k-l}\right\} ;\left\{\Xi^{k, p-1-k,-1}\right\},\left\{\Upsilon^{k, p-2-k,-1}\right\}\right)$ interpolating the cointertwiners, i.e. fulfilling (2.2.7)-(2.2.12).

Here is a sketch of the proof. As $\mathcal{O}(1) \cup \mathcal{O}(2)$ is a good covering of the pair $X, Y$, the cohomology isomorphism (1.4.3) holds true. Set

$$
\begin{aligned}
& \Xi^{-1,-1, p}=\Xi_{(1)}^{-1,-1, p}=\Xi_{(2)}^{-1,-1, p}, \\
& \Upsilon^{-1,-1, p-1}=\Upsilon_{(1)}^{-1,-1, p-1}=\Upsilon_{(2)}^{-1,-1, p-1} .
\end{aligned}
$$

Then, $\left(\Xi^{-1,-1, p}, \Upsilon^{-1,-1, p-1}\right)$ is a relative de Rham $p$-cocycle,

$$
\begin{aligned}
& d \Xi^{-1,-1, p}=0, \\
& i^{*} \Xi^{-1,-1, p}-d \Upsilon^{-1,-1, p-1}=0 .
\end{aligned}
$$

This can be extended to an $\mathcal{O}(1) \cup \mathcal{O}(2)$ relative cointertwiner, which is precisely the sequence of cochains interpolating the given relative Čech-de Rham cointertwiners we are looking for. Indeed, (2.2.7)-(2.2.9) are nothing but the transcription of (1.2.17)-(1.2.19) for the covering $\mathcal{O}(1) \cup \mathcal{O}(2)$. One verifies that relations (2.2.10)-(2.2.12) define two relative Cech-de Rham $p$-cointertwiner extending $\left(\Xi_{(1)}^{-1,-1, p}, \Upsilon_{(1)}^{-1,-1, p-1}\right),\left(\Xi_{(2)}^{-1,-1, p}, \Upsilon_{(2)}^{-1,-1, p-1}\right)$. 
Thus, these cointertwiners must equal the original cointertwiners up to trivial shifts preserving $\left(\Xi_{(1)}^{-1,-1, p}, \Upsilon_{(1)}^{-1,-1, p-1}\right),\left(\Xi_{(2)}^{-1,-1, p}, \Upsilon_{(2)}^{-1,-1, p-1}\right)$ (see the discussion of subsect. 1.4). If the relative de Rham cocycles $\left(\Xi_{(1)}^{-1,-1, p}, \Upsilon_{(1)}^{-1,-1, p-1}\right),\left(\Xi_{(2)}^{-1,-1, p}, \Upsilon_{(2)}^{-1,-1, p-1}\right)$ are cohomologically integer, the relative real Čech cocycles $\left(\Xi_{(1)}^{p,-1,-1}, \Upsilon_{(1)}^{p-1,-1,-1}\right),\left(\Xi_{(2)}^{-1, p,-1}\right.$, $\Upsilon_{(2)}^{-1, p-1,-1}$ ) are also cohomologically integer (cfr. subsect. 1.5) and, therefore, fit into two relative differential $p$-cocycles $\left(\Xi_{(1)}^{p,-1,-1}, \Upsilon_{(1)}^{p-1,-1,-1} ; \Xi_{(1)}^{* p-1,-1,-1}, \Upsilon_{(1)}^{* p-2,-1,-1} ; \hat{\Xi}_{(1)}^{p,-1,-1}\right.$, $\left.\hat{\Upsilon}_{(1)}^{p-1,-1,-1}\right),\left(\Xi_{(2)}^{-1, p,-1}, \Upsilon_{(2)}^{-1, p-1,-1} ; \Xi_{(2)}^{*-1, p-1,-1}, \Upsilon_{(2)}^{*-1, p-2,-1} ; \hat{\Xi}_{(2)}^{-1, p,-1}, \hat{\Upsilon}_{(2)}^{-1, p-1,-1}\right)(\mathrm{cfr}$. eqs. (1.3.1)-(1.3.3)). In that case, $\left(\left\{\Xi^{k, p-1-k,-1}\right\},\left\{\Upsilon^{k, p-2-k,-1}\right\}\right)$ extends to a sequence of cochains $\left(\left\{\Xi^{k, p-1-k,-1}\right\},\left\{\Upsilon^{k, p-2-k,-1}\right\} ;\left\{\Xi^{* k, p-2-k,-1}\right\},\left\{\Upsilon^{* k, p-3-k,-1}\right\} ;\left\{\hat{\Xi}^{k, p-1-k,-1}\right\}\right.$, $\left.\left\{\hat{\Upsilon}^{k, p-2-k,-1}\right\}\right)$ interpolating those cocycles, i.e. satisfying (2.2.21)-(2.2.26), after possibly shifting the latter by torsion differential cocycles (cfr. eqs. (1.3.4)-(1.3.6)) preserving $\left(\Xi_{(1)}^{p,-1,-1}, \Upsilon_{(1)}^{p-1,-1,-1}\right),\left(\Xi_{(2)}^{-1, p,-1}, \Upsilon_{(2)}^{-1, p-1,-1}\right)$. Moreover, when the relative integer Čech cocycles $\left(\hat{\Xi}_{(1)}^{p,-1,-1}, \hat{\Upsilon}_{(1)}^{p-1,-1,-1}\right),\left(\hat{\Xi}_{(2)}^{-1, p,-1}, \hat{\Upsilon}_{(2)}^{-1, p-1,-1}\right)$ are representatives of the same relative integer singular cohomology class via the isomorphism (1.5.1), the shifts by torsion differential cocycles preserve that cohomology class.

Indeed, the relative de Rham cocycle $\left(\Xi^{-1,-1, p}, \Upsilon^{-1,-1, p-1}\right)$ is cohomologically integer, so that the relative real Cech cocycle $\left(\left\{\Xi^{k, p-1-k,-1}\right\},\left\{\Upsilon^{k, p-2-k,-1}\right\}\right)$ is similarly cohomologically integer. Thus, it can be extended to an $\mathcal{O}(1) \cup \mathcal{O}(2)$ relative differential cocycle, which is the desired interpolating sequence of cochains. (2.2.21)-(2.2.23) are indeed the transcription of (1.3.1)-(1.3.3) for the covering $\mathcal{O}(1) \cup \mathcal{O}(2)$. One then checks that relations (2.2.24)-(2.2.26) define two relative differential $p$-cocycles extending $\left(\Xi_{(1)}^{p,-1,-1}, \Upsilon_{(1)}^{p-1,-1,-1}\right),\left(\Xi_{(2)}^{-1, p,-1}, \Upsilon_{(2)}^{-1, p-1,-1}\right)$. Thus, they must equal the given relative differential cocycles up to a torsion differential cocycle preserving $\left(\Xi_{(1)}^{p,-1,-1}, \Upsilon_{(1)}^{p-1,-1,-1}\right)$, $\left(\Xi_{(2)}^{-1, p,-1}, \Upsilon_{(2)}^{-1, p-1,-1}\right)$. When the relative integer Čech cocycles $\left(\hat{\Xi}_{(1)}^{p,-1,-1}, \hat{\Upsilon}_{(1)}^{p-1,-1,-1}\right)$, $\left(\hat{\Xi}_{(2)}^{-1, p,-1}, \hat{\Upsilon}_{(2)}^{-1, p-1,-1}\right)$ are representatives of the same relative integer singular cohomology class, the interpolating sequence of cochains can be chosen so that the relative integer Čech cocycle $\left(\left\{\hat{\Xi}^{k, p-1-k,-1}\right\},\left\{\hat{\Upsilon}^{k, p-2-k,-1}\right\}\right)$ is also a representative of that cohomology class. In that instance, relations (2.2.26) define two relative integer Čech cocycles representing again that cohomology class and thus equivalent to $\left(\hat{\Xi}_{(1)}^{p,-1,-1}, \hat{\Upsilon}_{(1)}^{p-1,-1,-1}\right)$, $\left(\hat{\Xi}_{(2)}^{-1, p,-1}, \hat{\Upsilon}_{(2)}^{-1, p-1,-1}\right)$ in relative integer Čech cohomology.

The above statements remain true if one of the two coverings, say $\mathcal{O}(2)$, is substituted by a refinement $\mathcal{O}^{\prime}(2)$ which is a good covering of $X, Y$ (cfr. subsects. 1.1, 1.4).

Indeed, as $\mathcal{O}^{\prime}(2)$ is a refinement of $\mathcal{O}(2)$, the associated refinement map $f_{2}$ induces a homomorphism $f_{2}{ }^{*}$ of the space relative Cech-de Rham cochains of $\mathcal{O}(1) \cup \mathcal{O}(2)$ into that 
of $\mathcal{O}(1) \cup \mathcal{O}^{\prime}(2)$, which preserves the de Rham and Čech degrees, commutes with $d$ and is such that $f_{2}{ }^{*} \delta_{1}=\delta_{1} f_{2}{ }^{*}, f_{2}{ }^{*} \delta_{2}=\delta^{\prime}{ }_{2} f_{2}{ }^{*}$. Then, sequence of cochains obtained by applying $f_{2}{ }^{*}$ to the interpolating sequence of cochains of $\mathcal{O}(1), \mathcal{O}(2)$ is interpolating with respect to $\mathcal{O}(1), \mathcal{O}^{\prime}(2)$.

It is easy to see that the above conditions on the coverings $\mathcal{O}(1), \mathcal{O}(2)$ are trivially satisfied for $\mathcal{O}(1)=\mathcal{O}(2)$, so that, in this special case, interpolating sequences of cochains exist. Then, interpolating sequences exist also when $\mathcal{O}(2)$ is a refinement of $\mathcal{O}(1)$.

In summary, we have shown the following.

First, there indeed exists a sequence of chains $\left(S_{-1,-1, p-1}, T_{-1,-1, p-2} ;\left\{V_{k, l, p-1-k-l}\right\}\right.$, $\left.\left\{Z_{k, l, p-2-k-l}\right\} ;\left\{S_{k, p-1-k,-1}\right\},\left\{T_{k, p-2-k,-1}\right\}\right)$ interpolating the intertwiners $\left(S_{-1,-1, p-1}^{(1)}\right.$, $\left.T_{-1,-1, p-2}^{(1)} ;\left\{V_{k,-1, p-1-k}^{(1)}\right\},\left\{Z_{k,-1, p-2-k}^{(1)}\right\} ; S_{p-1,-1,-1}^{(1)}, T_{p-2,-1,-1}^{(1)}\right),\left(S_{-1,-1, p-1}^{(2)}, T_{-1,-1, p-2}^{(2)}\right.$; $\left.\left\{V_{-1, k, p-1-k}^{(2)}\right\},\left\{Z_{-1, k, p-2-k}^{(2)}\right\} ; S_{-1, p-1,-1}^{(2)}, T_{-1, p-2,-1}^{(2)}\right)$ such that the sequence of chains $\left(\left\{S_{k, p-1-k,-1}\right\},\left\{T_{k, p-2-k,-1}\right\}\right)$ interpolates the integer Čech cycles $\left(S_{p-1,-1,-1}^{(1)}, T_{p-2,-1,-1}^{(1)}\right)$, $\left(S_{-1, p-1,-1}^{(2)}, T_{-1, p-2,-1}^{(2)}\right)$, possibly after shifting the intertwiners by trivial intertwiners leav$\operatorname{ing}\left(S_{-1,-1, p-1}^{(1)}, T_{-1,-1, p-2}^{(1)}\right),\left(S_{-1,-1, p-1}^{(2)}, T_{-1,-1, p-2}^{(2)}\right)$ unchanged.

Second, provided the good coverings $\mathcal{O}(1), \mathcal{O}(2)$ satisfy the conditions illustrated above, there indeed exist a sequence of cochains $\left(\Xi^{-1,-1, p}, \Upsilon^{-1,-1, p-1} ;\left\{\Omega^{k, l, p-2-k-l}\right\}\right.$, $\left.\left\{\Theta^{k, l, p-3-k-l}\right\} ;\left\{\Xi^{k, p-1-k,-1}\right\},\left\{\Upsilon^{k, p-2-k,-1}\right\}\right)$ interpolating the cointertwiners $\left(\Xi_{(1)}^{-1,-1, p}\right.$, $\left.\Upsilon_{(1)}^{-1,-1, p-1} ;\left\{\Omega_{(1)}^{k,-1, p-1-k}\right\},\left\{\Theta_{(1)}^{k,-1, p-2-k}\right\} ; \Xi_{(1)}^{p,-1,-1}, \Upsilon_{(1)}^{p-1,-1,-1}\right),\left(\Xi_{(2)}^{-1,-1, p}, \Upsilon_{(2)}^{-1,-1, p-1}\right.$; $\left.\left\{\Omega_{(2)}^{-1, k, p-1-k}\right\},\left\{\Theta_{(2)}^{-1, k, p-2-k}\right\} ; \Xi_{(2)}^{-1, p,-1}, \Upsilon_{(2)}^{-1, p-1,-1}\right)$ and a sequence of cochains $\left(\left\{\Xi^{k, p-1-k,}\right.\right.$ $\left.\left.{ }^{-1}\right\},\left\{\Upsilon^{k, p-2-k,-1}\right\} ;\left\{\Xi^{* k, p-2-k,-1}\right\},\left\{\Upsilon^{* k, p-3-k,-1}\right\} ;\left\{\hat{\Xi}^{k, p-1-k,-1}\right\},\left\{\hat{\Upsilon}^{k, p-2-k,-1}\right\}\right)$ interpolating the differential cocycles $\left(\Xi_{(1)}^{p,-1,-1}, \Upsilon_{(1)}^{p-1,-1,-1} ; \Xi_{(1)}^{* p-1,-1,-1}, \Upsilon_{(1)}^{* p-2,-1,-1} ; \hat{\Xi}_{(1)}^{p,-1,-1}\right.$, $\left.\hat{\Upsilon}_{(1)}^{p-1,-1,-1}\right),\left(\Xi_{(2)}^{-1, p,-1}, \Upsilon_{(2)}^{-1, p-1,-1} ; \Xi_{(2)}^{*-1, p-1,-1}, \Upsilon_{(2)}^{*-1, p-2,-1} ; \hat{\Xi}_{(2)}^{-1, p,-1}, \hat{\Upsilon}_{(2)}^{-1, p-1,-1}\right)$, which are compatible in the sense that the end of the first interpolating sequence equals the beginning of the second, as shown by the notation, possibly after shifting the cointertwiners and the differential cocycles by trivial cointertwiners and torsion differential cocycles leaving $\left(\Xi_{(1)}^{-1,-1, p}, \Upsilon_{(1)}^{-1,-1, p-1}\right),\left(\Xi_{(2)}^{-1,-1, p}, \Upsilon_{(2)}^{-1,-1, p-1}\right)$ unchanged. Further, when the relative integer Čech cocycles $\left(\hat{\Xi}_{(1)}^{p,-1,-1}, \hat{\Upsilon}_{(1)}^{p-1,-1,-1}\right),\left(\hat{\Xi}_{(2)}^{-1, p,-1}, \hat{\Upsilon}_{(2)}^{-1, p-1,-1}\right)$ represent of the same relative integer singular cohomology class, the shifts by torsion differential cocycles preserve that cohomology class.

Then, by (2.1.7), (2.2.18), (2.2.31), for given $\left(S_{p-1}, T_{p-2}\right) \in Z_{p-1}^{s \mathcal{O}(i)}(X, Y), i=1,2$, $\left(\Xi^{p}, \Upsilon^{p-1}\right) \in Z_{d R \mathbb{Z}}^{p}(X, Y)$, the difference $I^{\mathcal{O}(2)}-I^{\mathcal{O}(1)}$ is integer, provided the relative data 
employed in the construction of $I^{\mathcal{O}(i)}$ are suitably chosen. Since, however, this may not be the case, we see that a weaker result holds in general, namely

$$
I^{\mathcal{O}(2)}+\Delta I^{\mathcal{O}(2)}-I^{\mathcal{O}(1)}-\Delta I^{\mathcal{O}(1)}=0 \bmod \mathbb{Z}
$$

where the indeterminations $\Delta I^{\mathcal{O}(i)}$, given by (2.1.8), account for the shifts relating the relative data used in $I^{\mathcal{O}(i)}$ and those for which the interpolating sequences exist.

As discussed in subsect. 2.1, the indeterminations $\Delta I^{\mathcal{O}(i)}$ are not integer in general. By demanding that $\left(\hat{\Xi}_{(1)}^{p,-1,-1}, \hat{\Upsilon}_{(1)}^{p-1,-1,-1}\right),\left(\hat{\Xi}_{(2)}^{-1, p,-1}, \hat{\Upsilon}_{(2)}^{-1, p-1,-1}\right)$ are representatives of a fixed cohomology class of $H_{s \mathbb{Z}}^{p}(X, Y)$ via (1.5.1), the $\Delta I^{\mathcal{O}(i)}$ are given modulo integers by expressions of the form of the right hand side of (2.1.11).

As we have seen in subsect. 2.1, for a given good covering $\mathcal{O}$, the $\mathbb{Z}$ linear functional $\Phi^{\mathcal{O}}: Z_{p-1}^{s \mathcal{O}}(X, Y) \rightarrow \mathbb{R} / \mathbb{Z}$, eq. (2.1.9), depends on a choice of a relative integer singular cohomology class in $H_{s \mathbb{Z}}^{p}(X, Y)$, a representative $\left(\Xi^{p}, \Upsilon^{p-1}\right) \in Z_{d R \mathbb{Z}}^{p}(X, Y)$ of the image of such class in $H_{d R \mathbb{Z}}^{p}(X, Y)$ and a point in the relative de Rham cohomology torus $H_{d R}^{p-1}(X, Y) / H_{d R \mathbb{Z}}^{p-1}(X, Y)$. The parametrization of the family of maps $\Phi^{\mathcal{O}}$ in terms of $H_{d R}^{p-1}(X, Y) / H_{d R \mathbb{Z}}^{p-1}(X, Y)$ is however not unique. A change of the parametrization changes $\Phi^{\mathcal{O}}$ by an amount given by (2.1.11), (2.1.12). Thus, after fixing the cohomology class in $H_{s \mathbb{Z}}^{p}(X, Y)$ and its representative $\left(\Xi^{p}, \Upsilon^{p-1}\right) \in Z_{d R \mathbb{Z}}^{p}(X, Y)$, there still is no natural way of comparing the maps $\Phi^{\mathcal{O}(1)}, \Phi^{\mathcal{O}(2)}$ for the good coverings $\mathcal{O}(1), \mathcal{O}(2)$, unless we have a mapping relating their $H_{d R}^{p-1}(X, Y) / H_{d R \mathbb{Z}}^{p-1}(X, Y)$ parametrizations. This is precisely the origin of the residual indetrminations $\Delta I^{\mathcal{O}(i)}$ of the previous paragraph.

Then, from (2.1.9) and (2.2.36), we can draw the following conclusions. Let $\mathcal{O}(1), \mathcal{O}(2)$ be two good coverings of $X, Y$ and $\mathcal{O}(12)$ be a common refinement of $\mathcal{O}(1), \mathcal{O}(2)$ which is also a good covering. Let $\left(S_{p-1}, T_{p-2}\right) \in Z_{p-1}^{s \mathcal{O}(12)}(X, Y),\left(\Xi^{p}, \Upsilon^{p-1}\right) \in Z_{d R \mathbb{Z}}^{p}(X, Y)$. The pairs of good coverings $\mathcal{O}(1), \mathcal{O}(12)$ and $\mathcal{O}(2), \mathcal{O}(12)$ satisfy the requirements sufficient for the existence of interpolating sequences of cochains. Then,

$$
\Phi^{\mathcal{O}(i)}(S, T ; \Xi, \Upsilon)=\Phi^{\mathcal{O}(12)}(S, T ; \Xi, \Upsilon), \quad i=1,2
$$

provided the $H_{d R}^{p-1}(X, Y) / H_{d R \mathbb{Z}}^{p-1}(X, Y)$ parametrization of $\Phi^{\mathcal{O}(i)}, \Phi^{\mathcal{O}(12)}$ is suitably chosen. Thus, for $\left(S_{p-1}, T_{p-2}\right) \in Z_{p-1}^{s \mathcal{O}(12)}(X, Y),\left(\Xi^{p}, \Upsilon^{p-1}\right) \in Z_{d R \mathbb{Z}}^{p}(X, Y)$,

$$
\Phi^{\mathcal{O}(1)}(S, T ; \Xi, \Upsilon)=\Phi^{\mathcal{O}(2)}(S, T ; \Xi, \Upsilon)
$$


provided the $H_{d R}^{p-1}(X, Y) / H_{d R \mathbb{Z}}^{p-1}(X, Y)$ parametrizations of $\Phi^{\mathcal{O}(1)}, \Phi^{\mathcal{O}(2)}$ are suitably chosen.

Let us assume that the family of good open coverings of $X, Y$ is cofinal in the family of open coverings of $X$ (cfr. subsects. 1.4, 1.5). The conditions under which this is the case will be analyzed separately. in appendix A1. Then, in the sense stated in (2.2.38), $\Phi^{\mathcal{O}}$ is independent from covering choices.

\section{Extension of $\Phi^{\mathcal{O}}$ to non $\mathcal{O}$-small relative cycles}

Since any dependence on a choice of open covering $\mathcal{O}$ is unnatural, we would like to extend the $\mathbb{Z}$ linear map $\Phi^{\mathcal{O}}: Z_{p-1}^{s \mathcal{O}}(X, Y) \rightarrow \mathbb{R} / \mathbb{Z}$ of subsect. 2.1 to a $\mathbb{Z}$ linear map $\Phi: Z_{p-1}^{s}(X, Y) \rightarrow \mathbb{R} / \mathbb{Z}$ independent from $\mathcal{O}$. This can indeed be done using the barycentric subdivision operator $q$ introduced in subsect. 1.1 as follows.

Let us fix the cohomology class of $H_{s \mathbb{Z}}^{p}(X, Y)$, its representative $\left(\Xi^{p}, \Upsilon^{p-1}\right) \in Z_{d R \mathbb{Z}}^{p}(X$, $Y$ ) and the point of the torus $H_{d R}^{p-1}(X, Y) / H_{d R \mathbb{Z}}^{p-1}(X, Y)$ involved in the definition of $\Phi^{\mathcal{O}}$. Let $\left(S_{p-1}, T_{p-2}\right) \in Z_{p-1}^{s}(X, Y)$ be a general relative singular $p-1$-cycle. Pick a good open covering $\mathcal{O}$ of the pair $X, Y$. For a sufficiently large $k \geq 0,\left(q^{k} S_{p-1}, q^{k} T_{p-2}\right) \in Z_{p-1}^{s \mathcal{O}}(X, Y)$ is $\mathcal{O}$-small. We then set

$$
\Phi(S, T ; \Xi, \Upsilon)=\Phi^{\mathcal{O}}\left(q^{k} S, q^{k} T ; \Xi, \Upsilon\right)
$$

Next, we shall show that the right hand side of (2.3.1) does not depend on $\mathcal{O}$ and $k$, making the definition well-posed.

From (1.1.5), for $k, l \geq 0$, one has

$$
b c^{(k, l)}+c^{(k, l)} b=q^{k}-q^{l}, \quad c^{(k, l)}=\operatorname{sgn}(k-l) \cdot c \sum_{r=\min (k, l)}^{\max (k, l)-1} q^{r}
$$

So, by $(1.2 .1)$

$$
\begin{aligned}
& b c^{(k, l)} S_{p-1}+i_{*} c^{(k, l)} T_{p-2}=q^{k} S_{p-1}-q^{l} S_{p-1}, \\
& b c^{(k, l)} T_{p-2}=q^{k} T_{p-2}-q^{l} T_{p-2} .
\end{aligned}
$$

Therefore, $\left(q^{k} S_{p-1}-q^{l} S_{p-1}, q^{k} T_{p-2}-q^{l} T_{p-2}\right)$ is the relative boundary of the relative chain $\left(c^{(k, l)} S_{p-1},-c^{(k, l)} T_{p-2}\right)$. Now, if $k, l$ are large enough, $q^{r} S_{p-1}, q^{r} T_{p-2}$ are both $\mathcal{O}$-small 
for $r \geq \min (k, l)$. Since $c$ preserves $\mathcal{O}$-smallness and the range of $c$ contains only degenerate chains (see subsect. 1.1), both $c^{(k, l)} S_{p-1}$ and $c^{(k, l)} T_{p-2}$ are $\mathcal{O}$-small and degenerate, by (2.3.2). Recall that degenerate chains are invisible, that is the integral of any form on any such chain vanishes. So, recalling (1.1.15), (2.1.10)

$$
\begin{aligned}
& \Phi^{\mathcal{O}}\left(q^{k} S, q^{k} T ; \Xi, \Upsilon\right)-\Phi^{\mathcal{O}}\left(q^{l} S, q^{l} T ; \Xi, \Upsilon\right) \\
= & \Phi^{\mathcal{O}}\left(q^{k} S-q^{l} S, q^{k} T-q^{l} T ; \Xi, \Upsilon\right) \\
= & \Phi^{\mathcal{O}}\left(b c^{(k, l)} S+i_{*} c^{(k, l)} T, b c^{(k, l)} T ; \Xi, \Upsilon\right) \\
= & \left\langle c^{(k, l)} S_{p-1}, \Xi^{p}\right\rangle+\left\langle c^{(k, l)} T_{p-2}, \Upsilon^{p-1}\right\rangle \\
= & 0, \quad \bmod \mathbb{Z} .
\end{aligned}
$$

This shows that the right hand side of (2.3.1) is independent from $k$

Let $\mathcal{O}(1), \mathcal{O}(2)$ be two good coverings. Let $\mathcal{O}(12)$ be a good covering refining both $\mathcal{O}(1), \mathcal{O}(2)$ and let $k$ be large enough so that $\left(q^{k} S_{p-1}, q^{k} T_{p-2}\right)$ is $\mathcal{O}(12)$-small. Then,

$$
\Phi^{\mathcal{O}(1)}\left(q^{k} S, q^{k} T ; \Xi, \Upsilon\right)-\Phi^{\mathcal{O}(2)}\left(q^{k} S, q^{k} T ; \Xi, \Upsilon\right)=0
$$

by (2.2.38), provided the $H_{d R}^{p-1}(X, Y) / H_{d R \mathbb{Z}}^{p-1}(X, Y)$ parametrizations of $\Phi^{\mathcal{O}(1)}, \Phi^{\mathcal{O}(2)}$ are suitably chosen. This shows that the right hand side of (2.3.1) is independent from $\mathcal{O}$.

We have thus managed to define a mapping $\Phi: Z_{p-1}^{s}(X, Y) \rightarrow \mathbb{R} / \mathbb{Z}$. It is easy to show that $\Phi$ is $\mathbb{Z}$ linear. For given $\mathbb{Z}$ linear combinations of relative cycles, one chooses a good covering $\mathcal{O}$ and a subdivision degree $k$ large enough so that all the relative cycles involved are $\mathcal{O}$-small. Then, the $\mathbb{Z}$ linearity of $\Phi$ follows trivially from that of $\Phi^{\mathcal{O}}$.

When the relative (co)cycles $\left(S_{p-1}, T_{p-2}\right),\left(\Xi^{p}, \Upsilon^{p-1}\right)$ are shifted by the relative (co)boundaries given by the right hand sides of (1.2.2), (1.2.14), respectively, one has

$$
\begin{aligned}
\Delta \Phi(S, T ; \Xi, \Upsilon)= & \left\langle S_{p-1}, \xi^{p-1}\right\rangle-\left\langle T_{p-2}, v^{p-2}\right\rangle \\
& +\left\langle s_{p}, \Xi^{p}\right\rangle-\left\langle t_{p-1}, \Upsilon^{p-1}\right\rangle \\
& +\left\langle s_{p}, d \xi^{p-1}\right\rangle-\left\langle t_{p-1}, i^{*} \xi^{p-1}-d v^{p-2}\right\rangle, \quad \bmod \mathbb{Z}
\end{aligned}
$$


Indeed, provided $k$ is large enough to make all chains involved $\mathcal{O}$-small, $\Delta \Phi(S, T ; \Xi, \Upsilon)=$ $\Delta \Phi^{\mathcal{O}}\left(q^{k} S, q^{k} T ; \Xi, \Upsilon\right)$, which, on account of (2.1.10), is given by

$$
\begin{aligned}
\Delta \Phi^{\mathcal{O}}\left(q^{k} S, q^{k} T ; \Xi, \Upsilon\right)= & \left\langle q^{k} S_{p-1}, \xi^{p-1}\right\rangle-\left\langle q^{k} T_{p-2}, v^{p-2}\right\rangle \\
& +\left\langle q^{k} s_{p}, \Xi^{p}\right\rangle-\left\langle q^{k} t_{p-1}, \Upsilon^{p-1}\right\rangle \\
& +\left\langle q^{k} s_{p}, d \xi^{p-1}\right\rangle-\left\langle q^{k} t_{p-1}, i^{*} \xi^{p-1}-d v^{p-2}\right\rangle, \quad \bmod \mathbb{Z}
\end{aligned}
$$

Using (2.3.2) and (1.2.1), one has

$$
\begin{aligned}
& b c^{(k, 0)} S_{p-1}+i_{*} c^{(k, 0)} T_{p-2}=q^{k} S_{p-1}-S_{p-1}, \\
& b c^{(k, 0)} T_{p-2}=q^{k} T_{p-2}-T_{p-2} . \\
& b c^{(k, 0)} s_{p}+c^{(k, 0)} b s_{p}=q^{k} s_{p}-s_{p} \\
& b c^{(k, 0)} t_{p-1}+c^{(k, 0)} b t_{p-1}=q^{k} t_{p-1}-t_{p-1} .
\end{aligned}
$$

As the range of $c$ contains only degenerate chains, $c^{(k, 0)} S_{p-1}, c^{(k, 0)} T_{p-2}, c^{(k, 0)} s_{p}, c^{(k, 0)} t_{p-1}$ are all degenerate, hence invisible. Then, by (2.3.8), the chains $q^{k} S_{p-1}-S_{p-1}, q^{k} T_{p-2}-$ $T_{p-2}, q^{k} s_{p}-s_{p}, q^{k} t_{p-1}-t_{p-1}$ are all invisible. It follows that the right hand side of (2.3.7) equals that of (2.3.6).

If we change the $H_{d R}^{p-1}(X, Y) / H_{d R \mathbb{Z}}^{p-1}(X, Y)$ parametrization, $\Phi(S, T ; \Xi, \Upsilon)$ varies of an amount given by

$$
\Delta \Phi(S, T ; \Xi, \Upsilon)=-\left[\left\langle S_{p-1}, \Pi^{p-1}\right\rangle-\left\langle T_{p-2}, \Sigma^{p-2}\right\rangle\right] \bmod \mathbb{Z}
$$

for some relative de Rham cocycle $\left(\Pi^{p-1}, \Sigma^{p-2}\right)$ defined up to cohomologically integer relative de Rham cocycles. Indeed, provided $k$ is so large that all chains involved are $\mathcal{O}$-small, $\Delta \Phi(S, T ; \Xi, \Upsilon)=\Delta \Phi^{\mathcal{O}}\left(q^{k} S, q^{k} T ; \Xi, \Upsilon\right)$, so that, by $(2.1 .12)$,

$$
\Delta \Phi^{\mathcal{O}}\left(q^{k} S, q^{k} T ; \Xi, \Upsilon\right)=-\left[\left\langle q^{k} S_{p-1}, \Pi^{p-1}\right\rangle-\left\langle q^{k} T_{p-2}, \Sigma^{p-2}\right\rangle\right] \bmod \mathbb{Z}
$$

By (2.3.8), the chains $q^{k} S_{p-1}-S_{p-1}, q^{k} T_{p-2}-T_{p-2}$, are all invisible. It follows that the right hand side of (2.3.9) equals that of (2.3.10). 
4. The end product: the family $\mathcal{C} \mathcal{S}_{X, Y}^{p}$ of relative Cheeger-Simons differential characters

We have thus defined a family of $\mathbb{Z}$ linear mapping $\Phi: Z_{p-1}^{s}(X, Y) \rightarrow \mathbb{R} / \mathbb{Z}$ parameterized by a relative integer singular cohomology class in $H_{s \mathbb{Z}}^{p}(X, Y)$, a representative $\left(\Xi^{p}, \Upsilon^{p-1}\right) \in Z_{d R \mathbb{Z}}^{p}(X, Y)$ of the image of such class in $H_{d R \mathbb{Z}}^{p}(X, Y)$ and a point in the relative de Rham cohomology torus $H_{d R}^{p-1}(X, Y) / H_{d R \mathbb{Z}}^{p-1}(X, Y)$. We claim that this is precisely the family of degree $p Y$ relative Cheeger-Simons differential character of $X, \mathcal{C S}_{X, Y}^{p}$. This will become clear in the next section. See also the heuristic discussion given in the introduction for comparison. It is important to recall that the above construction works provided the family of good open coverings of $X, Y$ is cofinal in the family of open coverings of $X$ (cfr. subsects. 1.4, 2.2).

\section{Formal properties of the relative Cheeger-Simons differential characters}

In this section, we shall define the relative Cheeger-Simons differential characters in abstract terms and study their main formal properties. This will lead us to identify the family $\mathcal{C S}_{X, Y}^{p}$ of these characters with the family of characters constructed in sect. 2 .

Let $p, X, Y$ satisfy the same assumptions as in subsect. 1.2.

\section{Basic properties of the relative Cheeger-Simons characters}

By definition, $\Phi \in \mathcal{C S}_{X, Y}^{p}$ if $\Phi: Z_{p-1}^{s}(X, Y) \rightarrow \mathbb{R} / \mathbb{Z}$ is a $\mathbb{Z}$ linear mapping and there is a relative de Rham $p$-cochain $\left(\Xi^{p}, \Upsilon^{p-1}\right) \in C_{d R}^{p}(X, Y)$ such that

$$
\Phi\left(b s-i_{*} t,-b t\right)=\left\langle s_{p}, \Xi^{p}\right\rangle-\left\langle t_{p-1}, \Upsilon^{p-1}\right\rangle \bmod \mathbb{Z},
$$

for all relative singular chains $\left(s_{p}, t_{p-1}\right) \in C_{p}^{s}(X, Y) . \mathcal{C} \mathcal{S}_{X, Y}^{p}$ is clearly a group.

Let $\Phi \in \mathcal{C S}_{X, Y}^{p}$. If $\left(s_{p}, t_{p-1}\right) \in Z_{p}^{s}(X, Y)$ is a relative singular $p$-cycle, then $\Phi(b s-$ $\left.i_{*} t,-b t\right)=0$, by (1.2.1) (with $p$ replaced by $p+1$ ). From (3.1.1), we thus get the quantization condition

$$
\left\langle s_{p}, \Xi^{p}\right\rangle-\left\langle t_{p-1}, \Upsilon^{p-1}\right\rangle \in \mathbb{Z}
$$

Further, if $\left(s_{p}, t_{p-1}\right) \in B_{p}^{s}(X, Y)$ is the boundary of a relative singular $p+1$-chain $\left(u_{p+1}, v_{p}\right)$, one has from $(3.1 .2)$

$$
\left\langle u_{p+1}, d \Xi^{p}\right\rangle-\left\langle v_{p}, i^{*} \Xi^{p}-d \Upsilon^{p-1}\right\rangle \in \mathbb{Z}
$$


by (1.2.2) (with $p$ replaced by $p+1)$ and (1.1.16), (1.1.17). By (3.1.3), since $\left(u_{p+1}, v_{p}\right)$ is arbitrary, $\left(\Xi^{p}, \Upsilon^{p-1}\right)$ must satisfy (1.2.13) and is thus a relative de Rham cocycle. From (3.1.2), $\left(\Xi^{p}, \Upsilon^{p-1}\right)$ is cohomologically integer. Therefore, for any $\Phi \in \mathcal{C} \mathcal{S}_{X, Y}^{p},\left(\Xi^{p}, \Upsilon^{p-1}\right) \in$ $Z_{d R \mathbb{Z}}^{p}(X, Y)$.

To any $\Phi \in \mathcal{C} \mathcal{S}_{X, Y}^{p}$ there is associated a well-defined relative integer singular cohomology class in $H_{s \mathbb{Z}}^{p}(X, Y)$ such that $\left(\Xi^{p}, \Upsilon^{p-1}\right)$ is a representative of the image of such class in $H_{d R \mathbb{Z}}^{p}(X, Y)$. Indeed, as $\mathbb{R} / \mathbb{Z}$ is a divisible group and $Z_{p-1}^{s}(X, Y)$ is a subgroup of the free group $C_{p-1}^{s}(X, Y)$, there is a $\mathbb{Z}$ linear mapping $\bar{\Phi}: C_{p-1}^{s}(X, Y) \rightarrow \mathbb{R}$ such that $\Phi=\bar{\Phi} \mid Z_{p-1}^{s}(X, Y) \bmod \mathbb{Z}$. Then, by (3.1.1),

$$
\Lambda^{p}\left(s_{p}\right)-\Gamma^{p-1}\left(t_{p-1}\right)=\bar{\Phi}\left(b s-i_{*} t,-b t\right)-\left\langle s_{p}, \Xi^{p}\right\rangle+\left\langle t_{p-1}, \Upsilon^{p-1}\right\rangle,
$$

with $\left(s_{p}, t_{p-1}\right) \in C_{p}^{s}(X, Y)$, defines a relative integer singular cochain $\left(\Lambda^{p}, \Gamma^{p-1}\right)$, which is readily checked to be a cocycle cohomologically equivalent to $\left(\Xi^{p}, \Upsilon^{p-1}\right)$. The choice of $\bar{\Phi}$ affects $\left(\Lambda^{p}, \Gamma^{p-1}\right)$ at most by a relative integer singular coboundary. Hence, the integer singular cohomology class of $\left(\Lambda^{p}, \Gamma^{p-1}\right)$ is unambiguously determined by $\Phi$.

Let $\left(\Pi^{p-1}, \Sigma^{p-2}\right) \in C_{d R}^{p-1}(X, Y)$ be a relative de Rham $p-1$-cochain. Then,

$$
\Phi(S, T)=\left\langle S_{p-1}, \Pi^{p-1}\right\rangle-\left\langle T_{p-2}, \Sigma^{p-2}\right\rangle \bmod \mathbb{Z},
$$

for $\left(S_{p-1}, T_{p-2}\right) \in Z_{p-1}^{s}(X, Y)$, defines a character $\Phi \in \mathcal{C S}_{X, Y}^{p}$. $\Phi$ depends only on the equivalence class of $\left(\Pi^{p-1}, \Sigma^{p-2}\right)$ modulo the cohomologically integer relative de Rham $p$ - 1-cocycles of $Z_{d R \mathbb{Z}}^{p-1}(X, Y)$. The class of $H_{s \mathbb{Z}}^{p}(X, Y)$ corresponding to $\Phi$ vanishes. The relative de Rham cocycle $\left(\Xi^{p}, \Upsilon^{p-1}\right)$ of $\Phi$ is the relative de Rham coboundary of $\left(\Pi^{p-1}, \Sigma^{p-2}\right)$ (cfr. eq. (1.2.14)) and $\left(\Xi^{p}, \Upsilon^{p-1}\right)$ vanishes in the important case when $\left(\Pi^{p-1}, \Sigma^{p-2}\right) \in Z_{d R}^{p-1}(X, Y)$.

\section{The first relative Cheeger-Simons exact sequence}

From the above discussion, it follows that there is an exact sequence of the form

$$
0 \rightarrow H_{d R}^{p-1}(X, Y) / H_{d R \mathbb{Z}}^{p-1}(X, Y) \rightarrow \mathcal{C} \mathcal{S}_{X, Y}^{p} \rightarrow A_{\mathbb{Z}}^{p}(X, Y) \rightarrow 0
$$

where $A_{\mathbb{Z}}^{p}(X, Y)$ is the subset of the Cartesian product $H_{s \mathbb{Z}}^{p}(X, Y) \times Z_{d R \mathbb{Z}}^{p}(X, Y)$ formed by the pairs of a relative integer singular cohomology class in $H_{s \mathbb{Z}}^{p}(X, Y)$ and a representative of the image of such class in $H_{d R \mathbb{Z}}^{p}(X, Y)$. The relative de Rham cohomology torus 
$H_{d R}^{p-1}(X, Y) / H_{d R \mathbb{Z}}^{p-1}(X, Y)$ appears here. It parameterizes the group of all $\Phi \in \mathcal{C S}_{X, Y}^{p}$ characterized by the same pair of data in $A_{\mathbb{Z}}^{p}(X, Y)$. The sequence (3.2.1) in the absolute case was found in ref. [32].

We note that the $\mathbb{Z}$ linear mappings $\Phi: Z_{p-1}^{s}(X, Y) \rightarrow \mathbb{R} / \mathbb{Z}$ constructed in sect. 2 all belong to $\mathcal{C S}_{X, Y}^{p}$ as they satisfy (3.1.1) on account of (2.3.6). Each such $\Phi$ is characterized by a relative integer singular cohomology class in $H_{s \mathbb{Z}}^{p}(X, Y)$ and a representative $\left(\Xi^{p}, \Upsilon^{p-1}\right) \in Z_{d R \mathbb{Z}}^{p}(X, Y)$ of the image of such class in $H_{d R \mathbb{Z}}^{p}(X, Y)$. As is easy to see, these relative data are precisely the ones defined abstractly in subsect. 3.1 above. Indeed, (3.1.4) is the statement in the language of singular cohomology that the sequence of cochains $\left(\Xi^{p}, \Upsilon^{p-1} ; \bar{\Phi} ; \Lambda^{p}, \Gamma^{p-1}\right)$ is a differential cocycles (cfr. subsect. 1.3 and the discussion of subsect. 2.1). The set of the $\Phi$ compatible with a fixed choice of the relative data is parameterized by $H_{d R}^{p-1}(X, Y) / H_{d R \mathbb{Z}}^{p-1}(X, Y)$. This justifies our claim that the family of $\mathbb{Z}$ linear mappings $\Phi$ of sect. 2 is precisely $\mathcal{C S}_{X, Y}^{p}$.

\section{The second relative Cheeger-Simons exact sequence}

From the above discussion, there is another exact sequence of the form

$$
0 \rightarrow C_{d R}^{p-1}(X, Y) / Z_{d R \mathbb{Z}}^{p-1}(X, Y) \rightarrow \mathcal{C S}_{X, Y}^{p} \rightarrow H_{s \mathbb{Z}}^{p}(X, Y) \rightarrow 0
$$

which is directly related to the first one. This sequence indicates that the group of all characters $\Phi \in \mathcal{C S}_{X, Y}^{p}$ characterized by the same cohomology class in $H_{s \mathbb{Z}}^{p}(X, Y)$ is isomorphic to $C_{d R}^{p-1}(X, Y) / Z_{d R \mathbb{Z}}^{p-1}(X, Y)$. In the absolute case, the sequence was found in ref. [11]. Its importance stems from the fact that it reveals the relation between the Cheeger-Simons differential characters and the smooth Beilinson-Deligne cohomology $[12,13,14]$.

The analysis of sect. 2 furnishes an expression of the depenedence of the CheegerSimons characters $\Phi \in \mathcal{C S}_{X, Y}^{p}$ on the cohomologically integer relative de Rham cocycle $\left(\Xi^{p}, \Upsilon^{p-1}\right) \in Z_{d R \mathbb{Z}}^{p}(X, Y)$ for a fixed class in $H_{s \mathbb{Z}}^{p}(X, Y)$. Indeed, from (2.3.6), if we shift $\left(\Xi^{p}, \Upsilon^{p-1}\right)$ by a relative de Rham coboundary of the form (1.2.14), $\Phi$ varies of an amount

$$
\Delta \Phi(S, T)=\left\langle S_{p-1}, \xi^{p-1}\right\rangle-\left\langle T_{p-2}, v^{p-2}\right\rangle
$$

for $\left(S_{p-1}, T_{p-2}\right) \in Z_{p-1}^{s}(X, Y)$. 


\section{Concluding remarks}

In this paper, we have shown that the proper treatment of the topological integrals appearing in many physical models such as gauge theory and string theory requires in an essential way relative (co)homology and leads to relative Cheeger-Simons differential characters. Instead of contenting ourselves with an abstract study of these matters, we have worked out a definition of relative Cheeger-Simons differential characters which is constructive, i. e. computable in principle, and which contains the ordinary CheegerSimons differential characters as a particular case. The resulting expressions are totally explicit and completely general and lend themselves also to a more formal type of study.

Our method relies heavily on Čech (co)homological machinery. This has its advantages and disadvantages. At any rate, it seems hardly avoidable when one has to deal with locally defined fields on arbitrary topologically non trivial manifolds. A major part of the effort consisted in showing independence from covering choices.

We limited ourselves to the case where the quantization conditions can be formulated in the framework of integral relative cohomology. This excludes interesting examples from $\mathrm{D}$-brane theory, which require more general cohomology theories such as $\mathrm{K}$ theory. It would be very interesting to generalize our constructions to $K$ theory. This is left for future work.

\section{A1. Existence and cofinality of good open coverings}

Let $M$ be a manifold equipped with a Riemannian metric $g$. For $m \in M$ and $u \in T_{m} M$, we set $|u|_{M g}=g_{m}(u, u)^{1 / 2}$. For $r>0$, we define $B_{M g}(m, r)=\left\{\left.u\left|u \in T_{m} M,\right| u\right|_{M g}<r\right\}$. The exponential $\exp _{M g}$ is a map of an open neighborhood $N_{M g}$ of the 0 section of $T M$ into $M$. It has the basic property that, for $m \in M$ and $u \in T_{m} M$, the curve $\gamma_{m u}(t)=$ $\exp _{M g}(t u), 0 \leq t, t u \in N_{M g}$, is the unique geodesic with initial condition $(m, u)$ [40].

The following theorem holds [40]. For $m \in M$, there is $r_{M g}(m)>0$ such that, for any $r$ with $0<r<r_{M g}(m), B_{M g}(m, r) \subseteq N_{M g}$ and there is an open neighborhood $U_{M g}(m, r)$ of $m$ in $M$ such that $\exp _{M g}: B_{M g}(m, r) \rightarrow U_{M g}(m, r)$ is a diffeomorphism. Further, $U_{M g}(m, r)$ is geodesically convex, that is every two points $p, q \in U_{M g}(m, r)$ can be joined by a unique distance minimizing geodesic contained in $U_{M g}(m, r)$. For $m \in M$, the family $\mathcal{U}_{M g}(m)=\left\{U_{M g}(m, r) \mid 0<r<r_{M g}(m)\right\}$ is a fundamental system of geodesically convex open neighborhoods of $m$. Since the intersection of any finite number of geodesically convex 
open sets is geodesically convex, the open coverings $\mathcal{O}$ of $M$ made of sets $U_{M g}(m, r)$ with varying $m$ and sufficiently small $r$ are good. Further, such good coverings are cofinal in the family of all open coverings (cfr. subsect. 1.1).

Let $X$ be a manifold equipped with a Riemannian metric $g$ and let $Y$ be a submanifold of $X$ with induced metric $i^{*} g$. Assume that $Y$ is totally geodesic [40]. Then, every geodesic of $Y$ with respect to the metric $i^{*} g$ is a geodesic of $X$ with respect to $g$, so that $\exp _{Y i^{*} g}=$ $\exp _{X g} \mid N_{Y i^{*} g} \cap N_{X g}$. It follows that for $y \in Y \subseteq X$ and $0<r<r_{Y i^{*} g}(y), U_{Y i^{*} g}(y, r)=$ $U_{X g}(y, r) \cap Y$. Now, define $\mathcal{U}_{X g}^{\prime}(x)=\left\{U_{X g}(x, r) \mid 0<r<r_{X g}(x), U_{X g}(x, r) \cap Y=\emptyset\right\}$, for $x \in X \backslash Y, \mathcal{U}_{X g}^{\prime}(y)=\left\{U_{X g}(y, r) \mid 0<r<r_{Y^{*} g}(x)\right\}$, for $y \in Y$. Then, for any $x \in X$, $\mathcal{U}_{X g}^{\prime}(x)$ is a fundamental system of geodesically convex open neighborhoods of $x$ such that, for any $y \in Y, \mathcal{U}_{X g}^{\prime}(y) \cap Y=\mathcal{U}_{Y i^{*} g}(y)$. From the discussion of the previous paragraph, it follows that the open coverings $\mathcal{O}$ of $X$ made of sets $U_{X g}(x, r)$ with varying $x$ and sufficiently small $r$ are good for the pair $X, Y$ (cfr. subsect. 1.4) and that such good coverings are cofinal in the family of all open coverings.

Therefore, given a manifold $X$ and a submanifold $Y$ of $X$, in order a cofinal family of good open coverings of $X, Y$ to exist, it is sufficient that there is a Riemannian metric $g$ on $X$ with respect to which $Y$ is totally geodesic.

Acknowledgements. We are greatly indebted to R. Stora for providing his invaluable experience and relevant literature.

\section{REFERENCES}

[1] O. Alvarez, "Cohomology And Field Theory", UCB-PTH-85/20 Plenary talk given at Symp. on Anomalies, Geometry and Topology, Argonne, IL, Mar 28-30, 1985.

[2] O. Alvarez, "Topological Quantization And Cohomology", Commun. Math. Phys. 100 (1985) 279.

[3] K. Gawedzki, "Topological Actions In Two-Dimensional Quantum Field Theories", in Cargese 1987, proceedings, Nonperturbative Quantum Field Theory, 101.

[4] D. S. Freed, "Locality and integration in topological field theory", hep-th/9209048.

[5] D. S. Freed, "Classical Chern-Simons theory. Part 1", Adv. Math. 113 (1995) 237 hep-th/9206021.

[6] D. I. Olive and M. Alvarez, "Spin and abelian electromagnetic duality on four-manifolds", Commun. Math. Phys. 217 (2001) 331, hep-th/0003155. 
[7] M. Alvarez and D. I. Olive, "The Dirac quantization condition for fluxes on fourmanifolds", Commun. Math. Phys. 210 (2000) 13, hep-th/9906093.

[8] M. Bauer, G. Girardi, R. Stora, F. Thuillier, "A class of topological actions", incomplete draft, June 2000.

[9] J. L. Koszul, "Travaux de S. S. Chern et J. Simons sur les classes caractéristiques" Seminaire Bourbaki 26ème année 440 (1973/74) 69.

[10] J. Cheeger, "Multiplication of Differential Characters", Convegno Geometrico INDAM, Roma maggio 1972, in Symposia Mathematica XI Academic Press (1973) 441.

[11] J. Cheeger and J, Simons, "Differential Characters and Geometric Invariants", Stony Brook preprint (1973) reprinted in Lecture Notes in Math. 1167 Springer Verlag (1985) 50.

[12] P. Deligne, "Théorie de Hodge", Inst. Hautes Études Sci. Publ. Math. 40 (1971) 5.

[13] A. A. Beilinson, "Higher regulators and values of L-functions", J. Soviet Math. 30 (1985) 2036.

[14] A. A. Beilinson, "Notes on absolute Hodge cohomology", in Contemp. Math. 55 Part I Amer. Math. Soc. (1986).

[15] M. J. Hopkins and I. M. Singer, "Quadratic Functions in Geometry, Topology, and M-Theory", incomplete draft, June 2000.

[16] D. S. Freed and E. Witten, "Anomalies in string theory with D-branes", hep-th/ 9907189 .

[17] A. Kapustin, "D-branes in a topologically nontrivial B-field", Adv. Theor. Math. Phys. 4 (2001) 127, hep-th/9909089.

[18] C. Bachas, M. Douglas and C. Schweigert, "Flux stabilization of D-branes", JHEP 0005 (2000) 048, hep-th/0003037.

[19] J. Polchinski, unpublished.

[20] W. Taylor, "D2-branes in B fields", JHEP 0007 (2000) 039, hep-th/0004141.

[21] A. Alekseev, A. Mironov and A. Morozov, "On B-independence of RR charges", Phys. Lett. B 532 (2002) 350, hep-th/0005244.

[22] S. Stanciu, "A note on D-branes in group manifolds: Flux quantization and D0charge", JHEP 0010 (2000) 015 hep-th/0006145.

[23] A. Alekseev and V. Schomerus, "RR charges of D2-branes in the WZW model", hepth/0007096.

[24] J. M. Figueroa-O'Farrill and S. Stanciu, "D-brane charge, flux quantization and relative (co)homology", JHEP 0101 (2001) 006, hep-th/0008038. 
[25] M. R. Douglas, "Topics in D-geometry", Class. Quant. Grav. 17 (2000) 1057, hepth/9910170.

[26] M. B. Green, J. A. Harvey and G. Moore, "I-brane inflow and anomalous couplings on D-branes", Class. Quant. Grav. 14 (1997) 47, hep-th/9605033.

[27] Y. E. Cheung and Z. Yin, Nucl. Phys. B517 (1998) 69, hep-th/9710206.

[28] R. Minasian and G. Moore, "K-theory and Ramond-Ramond charge", JHEP 9711 (1997) 002, hep-th/9710230.

[29] E. Witten, "D-branes and K-theory", JHEP 9812 (1998) 019, hep-th/9810188.

[30] E. Witten, "Overview of K-theory applied to strings", Int. J. Mod. Phys. A 16 (2001) 693, hep-th/0007175.

[31] G. Moore and E. Witten, "Self-duality, Ramond-Ramond fields, and K-theory", JHEP 0005 (2000) 032, hep-th/9912279.

$[32]$ D. S. Freed and M. J. Hopkins, "On Ramond-Ramond fields and K-theory", JHEP 0005 (2000) 044, hep-th/0002027.

[33] R. Gopakumar, S. Minwalla, N. Seiberg and A. Strominger, "OM theory in diverse dimensions", JHEP 0008 (2000) 008, hep-th/0006062.

[34] R. Bott and L. Tu, "Differential Forms in Algebraic Topology", Springer Verlag, New York, 1982.

[35] M. Karoubi and C. Leruste, "Algebraic Topology via Differential Geometry", Cambridge University Press, Cambridge, 1987.

[36] J. W. Vick, "Homology Theory", Academic Press, New York and London, 1973.

[37] J. -L. Brylinski, "Loop Spaces, Characteristic Classes and Geometric Quantization", Birkhäuser, 1993.

[38] N. Hitchin, "Lectures on special Lagrangean submanifolds", math.DG/9907034.

[39] A. Weil, "Sur les théorèmes de de Rham", Commen. Math. Helv. 26 (1952) 17.

[40] S. Kobayashi and K. Nomizu, "Foundations of Differential Geometry", vols. I and II, J. Wiley \& Sons (1963). 